\title{
Angle and Time of Arrival Characteristics of 3D Air-to-Ground Radio Propagation Environments
}

\author{
Sardar Muhammad Gulfam*, Syed Junaid Nawaz ${ }^{*+}$, Abrar Ahmed*, \\ and Mohammad N. Patwary ${ }^{\S}$. \\ *Department of Electrical Engineering, COMSATS Institute of Information \\ Technology, Islamabad, Pakistan.

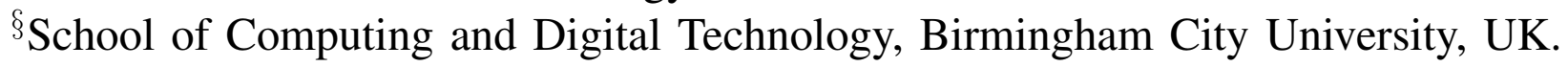 \\ sardar_muhammad@comsats.edu.pk, junaidnawaz@ieee.org, \\ abrar_ahmed@comsats.edu.pk, and mohammad.patwary@bcu.ac.uk
}

\begin{abstract}
A three dimensional (3D) geometric channel model is proposed for ground-to-air (G2A) and air-toground (A2G) communication links. A low-elevated ground station (GS) and a high-elevated air station (AS) are taken at foci points of a virtual bounding ellipsoid corresponded from known knowledge of delay of longest propagation path. The effective region of scatterers around the GS is designed on the basis of this ellipsoid truncated by the average rooftop level (or average height of sea waves) and ground plane. Closedform expressions for joint and marginal probability density functions (PDFs) of angle of arrival (AoA) observed at AS and GS in correspondence with azimuth and elevation angles are derived. Furthermore, closed-form expressions for density of energy with respect to the delay of arriving multipath waves corresponded from both the elevation and azimuth AoA are derived independently when observed from either end of the communication link. Moreover, effect of different physical parameters of the channel on distribution of energy in angular and temporal domains is presented. The comparison of analytical results with results of a notable model is also presented. In order to verify the derived analytical expressions, a comparison of analytical results with the performed simulation results is presented, which shows a good match.
\end{abstract}

Keywords: Channel Modeling, Air-to-Ground Communication, Ellipsoid, Multipath Channels, Scattering. 


\section{INTRODUCTION}

Mobile communications and internet access are increasingly becoming an essential part of people's lives in today's information society. This has led to the demands of users for high speed, reliable, low cost and all-time network connectivity. As the technology of providing network coverage to densely populated users has gained significant advancement, the demand of high speed internet in aircrafts has also increased drastically. A long range, high speed, and reliable link is not only needed for provision of data services to passengers, but it is also of great importance for provision of security, navigation and other services to aeroplanes. Airborne communication applications are not only limited to the scenarios for communication over the ground surface; however, various busy air routes of aircrafts across the remote ocean regions raise the importance of studying the communication characteristics over the sea surface. The airborne platform can also be used as an alternative of traditional terrestrial communication systems for providing data and voice services over large geographic regions; i.e., high-altitude platform (HAP) for wireless communications [1,2]. The advantages of using HAP is cost effective coverage of rural and maritime regions and system flexibility. The possibility of upgrading the platform also reduces the risk of technology obsolescence. Applications of ground/sea-to-air (G2A) and air-to-ground/sea (A2G) communications links include link of HAPs and unmanned air vehicle (UAV) with low altitude nodes at ground stations (GSs) for different purposes; viz: sea lane monitoring, law enforcement, industrial inspection, emergency humanitarian missions, broadcast HDTV signal, high-speed wireless access, navigation and position location systems, intelligent transportation systems, surveillance, military applications and large scale temporary events.

To meet the dynamic demands of $\mathrm{A} 2 \mathrm{G}$ and $\mathrm{G} 2 \mathrm{~A}$ communications systems, the study on different characteristics for A2G channel is very important. Accurate knowledge of the behavior of the channel at nodes of communication link has an important role in reliable communication systems; therefore, various studies on modeling the channel's characteristics for terrestrial communications links has been proposed in the literature [3-11]. Similarly, it is required to conduct a study for understanding channel's behavior in A2G/G2A scenarios. In this regard, A2G/G2A communication scenarios have been thoroughly investigated in the literature. Various methods for provision of coverage in aeroplanes from GSs (i.e., A2G/G2A) are proposed by the researchers. Modeling and 
characterization of G2A/A2G channels on the basis of measurement campaigns are given in [1219]. A comprehensive review of channels models for $A 2 \mathrm{G} / \mathrm{G} 2 \mathrm{~A}$ radio propagation environments is provided in [12]. Research works on modeling and characterization of A2G channels over earth surface (mountains, deserts, etc) are provided in $[14,15,18]$. In [15], a study based on measurement campaigns on wideband channel characterization is presented using antenna arrays with low altitudes at $2 \mathrm{GHz}$. In [18], a channel model is proposed based on measurements for wideband aeronautical communication links. For the scenario of A2G links over sea surface, few studies are available in the literature (e.g., $[16,17,20])$ for characterization of the channel. The studies in [16] and [17] are based on measurements and limited for $\mathrm{C}$ band. Another work on the modeling of a multipath propagation channel over sea surface is given in [20]. The proposed solution depicts that a 3-ray multipath model consisting of a line-of-sight (LoS) path and two reflected propagation paths provides a good fit to the measured channel responses. However, it considers only 3 multipath components but there is higher probability for many multipath components when airborne altitude decreases. The recent work on A2G/G2A MIMO channels is provided in [21] where measurements are given for a specific scenario where aircraft travel in a loop. The Root Mean Square (RMS) Delay Spread (DS) is analyzed and cosine Hermitian angle between channel vectors is computed. The authors discussed the effects of roll angle of the aircraft. Global coverage in aeronautical communication networks can be achieved through a satellite node. Therefore, various studies for such aircraft-to-satellite and satellite-to-ground communication links are available in the literature (e.g., $[19,22])$. The main advantage of satellite links global coverage however this link has many disadvantagesas well, such as: high cost and long delay. The challenge of global coverage provision for aircrafts flying over remote regions can now be addressed through advanced mesh networks based and direct A2G links based techniques [22,23], which avoid the use of satellite links.

Various notable contributions are found in the literature on the concept of providing wireless communication system through HAP [1,2, 24-27]. In [1], many advantages of HAP over terrestrial and satellite based systems are discussed. In [2], authors have proposed a HAP based system for broadband wireless metropolitan area network with a star topology whose solitary hub is located in the atmosphere. The role of HAP based systems in providing global connectivity for future systems 
and services is discussed in [24]. The authors discussed constraints of HAPs and established that goal of global connectivity can be achieved by the integration of terrestrial, HAPs, and satellite networks. The research is very active in connection with designing and usage of HAP; however, less work is available on channel characteristics of HAP communication systems. The channel characteristics like power delay profile and Doppler spectrum for HAP based system are analyzed in [25]. A channel model for HAP-MIMO is given in [27] and space-time correlation function is derived. A geometric channel model for HAP cellular communication scenarios is presented in [26]; however, in this study, HAP elevation angle is not considered and it only provides an analysis on power delay profiles and coherence bandwith. Moreover, work on angular characteristics of the channel for HAP based systems is absent in the literature.

Accurate understanding of temporal and spatial characteristics of channel is very important for performance evaluation of communication links. These spatial characteristics are used for calculating different shape factors (i.e., delay spread, angular spread etc). Geometric channel models are the famous solutions used to model the temporal and spatial characteristics. Geometric channel models provide accuracy in modeling the channel's behavior by giving a probabilistic spatial relation among receiver, scatterer, and transmitter. A number of models are proposed based on geometry for the characterization of temporal and spatial domain of channel. The joint and marginal probability density functions (PDFs) of time-of-arrival (ToA) and angle-of-arrival (AoA) are derived for two dimensional (2D) circular and elliptical models in [28] and [5], respectively. A geometry based analysis on A2G channel correlation matrix for UAV MIMO communication systems is presented in [29]. A study on Doppler spectrum and delay characterization of A2G channels is presented in [30]. A notable work on modeling of A2G channels is given in [31] for non selective and selective behavior of A2G multipath scenarios. A geometric A2G channel model is proposed for studying spatial characteristics by Newhall et al. in [32]. The author derived expressions for PDF of AoA and delay by assuming single bounce propagation. The scattering region is taken as 2D shaped around GS; however, propagation from high rise structures (like mountains and buildings etc) is ignored. Another geometric model for UHF band is proposed in [33] . The above discussed geometry based studies on modeling of A2G/G2A communications are only applicable for communication over ground surface. Moreover, 2D models have advantages 
like less computational complexity of over three dimensional (3D) models, it is proven in the literature $[3,8,34]$ that considering propagation in elevation plane is important for modeling a propagation environment realistically. Such work for A2G/G2A communication scenarios on 3D geometric channel modeling is missing in the literature. Nevertheless, there is scope to develop a more realistic $3 \mathrm{D}$ model for $\mathrm{G} 2 \mathrm{~A}$ and $\mathrm{A} 2 \mathrm{G}$ propagation environment to study the channel characteristics.

In this paper, a novel 3D geometric channel model for characterization of $\mathrm{G} 2 \mathrm{~A} / \mathrm{A} 2 \mathrm{G}$ propagation scenarios is proposed. A part of the initial findings of this work is presented in [35], where only spatial statistics of the radio channel are discussed. The model proposed in this paper is defined for both spatial and temporal characteristics of the radio channel observed at both ends of the link with an improved definition of the effective scattering region making the model applicable for communication over both ground and sea surface. The proposed model considers bounding ellipsoid corresponded from delay of longest propagation path where GS and air station (AS) assumed at foci points of the ellipsoid. The shape of scattering region is obtained by truncating this bounding ellipsoid upto a level of equal to average height of rooftop or average height of sea-wave in the GS's vicinity. Closed-form expressions are derived at both the nodes of link for marginal and joint PDF of elevation and azimuth AoA and ToA. The impact of physical parameters of the channel on AoA and ToA statistics is studied thoroughly. The organisation of the paper is as follows: The proposed 3D propagation model is discussed in Section II. Section III discusses the derivation of joint and marginal PDFs of AoA observed at AS and GS for elevation and azimuth angles along with simulated and theoretical results. Section IV discusses the joint and marginal PDFs for ToA of the multipath, corresponded with elevation and azimuth angles for the proposed model along with theoretical results. Finally, Section V discusses the conclusions on the obtained results.

\section{System Model}

In this section, the details of $3 \mathrm{D}$ channel model are presented which is proposed for $\mathrm{G} 2 \mathrm{~A} / \mathrm{A} 2 \mathrm{G}$ propagation scenarios. The proposed model is represented in Fig. 1, where GS and AS are taken at heights $h_{\mathrm{GS}}$ and $h_{\mathrm{AS}}$, respectively. In case of aeronautical communication scenarios, AS may be an aircraft and GS may be a ground base station serving the aircraft. However, in the case 


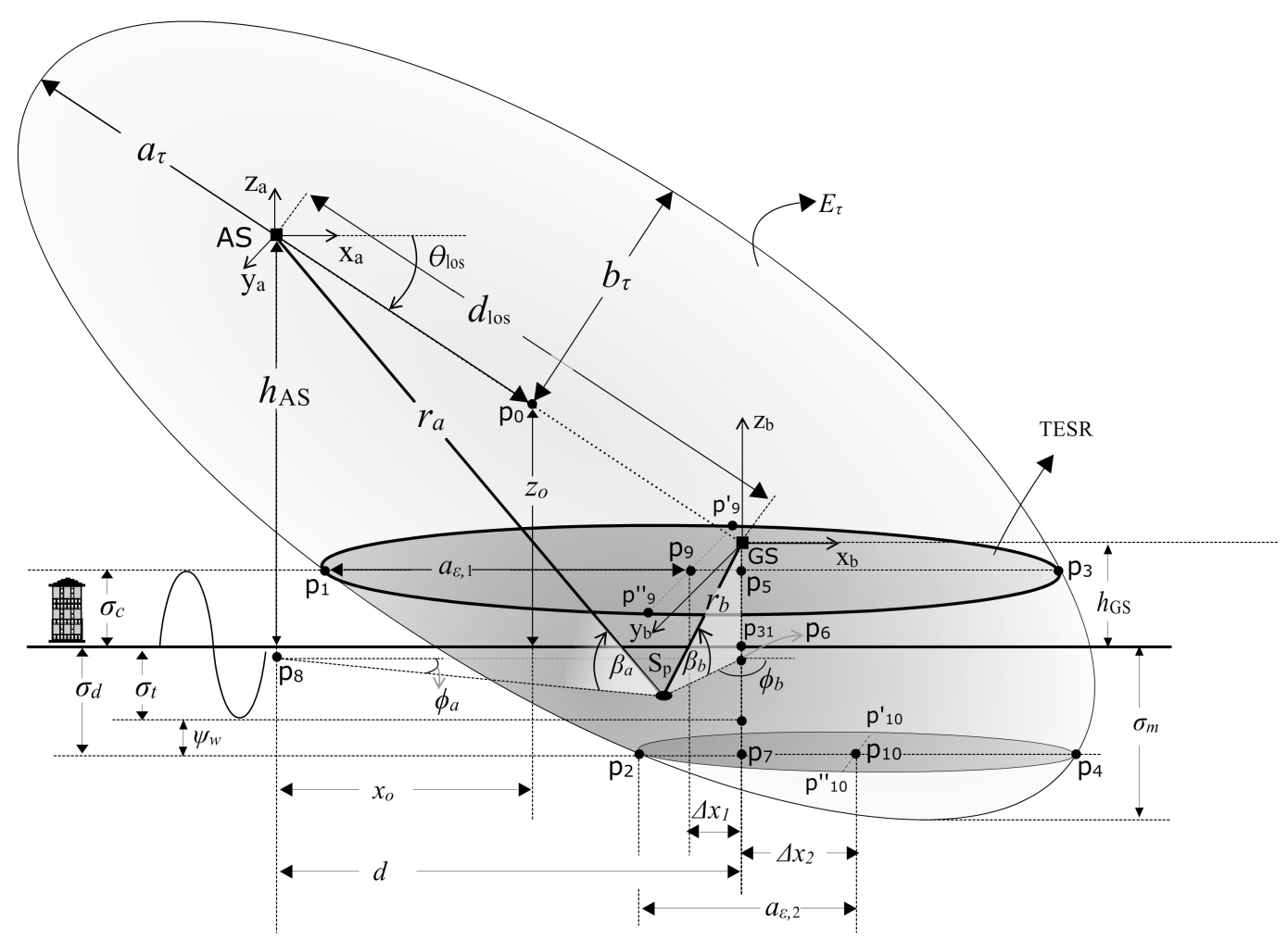

Fig. 1. Geometry of 3D channel model proposed for G2A/A2G communication scenarios.

of HAP cellular communication scenarios, the AS and GS may be a high-elevated base station and a mobile user on ground surface, respectively. For such dynamic scenarios of A2G radio communications, it becomes important to comprehensively study the channel characteristics seen at both the nodes. The GS and AS are separated by distance $d$ from each other. The line-of-sight (LoS) distance from GS to AS is represented by $d_{\text {los }}$, which can be expressed as,

$$
d_{\mathrm{los}}=\sqrt{d^{2}+\left(h_{\mathrm{AS}}-h_{\mathrm{GS}}\right)^{2}} .
$$

The inclination angle (which is referred as an elevation angle in this manuscript) of LoS component with ground plane is $\theta_{\text {los }}$,

$$
\theta_{\mathrm{los}}=\arctan \left(\frac{h_{\mathrm{AS}}-h_{\mathrm{GS}}}{d}\right)
$$

The sum of distances from two foci points of an (oblate) ellipsoid to any point on its surface is constant, regardless of the point on the surface. Using this property, the boundary of scattering 
region can be drawn by the ellipsoid $\left(E_{\tau}\right)$ corresponded from the multipath component with longest delay, with it's foci points at AS and GS. The minor, intermediate, and major dimensions of this ellipsoid $\left(E_{\tau}\right)$ can thus be calculated from longest path's delay $\left(\tau_{\max }\right)$, given as below,

$$
\begin{gathered}
a_{\tau}=\frac{c \tau_{\max }}{2}, \\
c_{\tau}=b_{\tau}=\sqrt{a_{\tau}^{2}-\left(\frac{d_{\text {los }}}{2}\right)^{2}} .
\end{gathered}
$$

Usually AS's height is more than the average rooftop level; therefore, the AS's vicinity is modeled as region without scatterers. The location GS can be on sea surface or at earth surface in various communication scenarios. In both cases, the GS's vicinity can be a rich scattering environment. For case of GS over sea, not only the sea surface but parts of a ship (or surfaces of an offshore platform) can also cause arrival of rich multipath components. The scattering region around GS is controlled by ellipsoid $\left(E_{\tau}\right)$ obtained by truncation of planes with height equal to average height of crest and trough of sea-waves or average rooftop level of surroundings, for cases of GS at sea surface or ground surface, respectively. Scatterers are taken only around GS confined inside truncated ellipsoidal shaped scattering region (TESR). A scatterer is assumed to scatter the incoming signal (from any direction) in all the directions with uniform random phases and equal scattering coefficients. The average height of trough and crest of sea-wave from mean level are represented with $\sigma_{t}$ and $\sigma_{c}$, respectively. In the communication scenario when GS is over ground surface, the value of $\sigma_{c}$ will be equal to average rooftop height. The behavior of propagation of electromagnetic (EM) waves in water medium is changed from that of propagation in air because of electrical conductivity and high permittivity. The major limit propagation of EM wave in water is the high attenuation of water [36]. The propagation loss and transmission loss in the water medium depends on properties of water, frequency, and incidence angle, etc. EM waves in low frequency range are observed to penetrate into the water upto a moderate depth. The model in this manuscript considers water penetration of EM waves for modeling of TESR. The penetration depth is shown by $\psi_{w}$. The penetration parameter can be set according to conditions of water and range of frequencies, and can be taken as zero to show no penetration (e.g., for higher frequencies). The penetration into the water and deviation of trough together shown the scattering region depth, which is shown by $\sigma_{d}$ (i.e., $\sigma_{d}=\sigma_{t}+\psi_{w}$ ) in Fig. 1. However maximum depth from 
mean level to sea bed is shown by $\sigma_{m}$. A 2D ellipse is formed at upper face of TESR whose minor and major axes are $b_{\epsilon 1}$ and $a_{\epsilon 1}$. Similarly, another 2D ellipse is formed at bottom face of TESR whose minor and major axes are $b_{\epsilon 2}$ and $a_{\epsilon 2}$. The center points of these $2 \mathrm{D}$ ellipses are shown by $\mathrm{p}_{9}$ and $\mathrm{p}_{10}$. The horizontal deviation of GS from the center points is represented by $\Delta x_{1}$ and $\Delta x_{2}$ (see Fig.1). In $\mathrm{x}-\mathrm{z}$ plane, a line is drawn parallel to $\mathrm{x}$-axis represents the rooftop level of TESR intersects the ellipsoid $E_{\tau}$ at points $\mathrm{p}_{1}$ and $\mathrm{p}_{3}$. The equation of the line can be substituted into equation of the ellipsoid $E_{\tau}$, the $x$-coordinate of the intersection points $\mathrm{p}_{1}$ and $\mathrm{p}_{3}$ can be obtained. Similarly, the $x$-coordinate of the intersection points $\mathrm{p}_{2}$ and $\mathrm{p}_{4}$ of the bounding ellipsoid $E_{\tau}$ and a line representing the ground level (or sea trough level plus penetration depth) can also be obtained. From calculated $x$-coordinate of intersection points, the major dimension $a_{\epsilon 2}$ and $a_{\epsilon 1}$ of the ellipses along x-axis formed at lower and upper face of TESR, can be obtained. After performing tedious simplifications, the combined generalized expression for $a_{\epsilon, n}$ (parameter $n=1$ and $n=2$ represents the upper and lower ellipse, respectively) is expressed as under,

$$
a_{\epsilon, n}=\frac{\sqrt{-2 a_{\tau}^{2} b_{\tau}^{2}\left(-a_{\tau}^{2}-b_{\tau}^{2}+2 \sigma_{n}^{2}-4 \sigma_{n} z_{o}+2 z_{o}^{2}+\left(a_{\tau}^{2}-b_{\tau}^{2}\right) \cos \left(2 \theta_{\mathrm{los}}\right)\right)}}{a_{\tau}^{2}+b_{\tau}^{2}+\left(-a_{\tau}^{2}+b_{\tau}^{2}\right) \cos \left(2 \theta_{\mathrm{los}}\right)} .
$$

Subsequently, the coordinates of the points $\mathrm{p}_{9}$ representing the origin of the upper face of TESR can be obtained from the calculated coordinates of the points $\mathrm{p}_{1}$ and $\mathrm{p}_{3}$. Substituting the equation of a line drawn parallel to y-axis in the horizontal 2D plane formed at upper face of TESR passing through the point $\mathrm{p}_{9}$, into the equation of the bounding ellipsoid $E_{\tau}$, the $y$-coordinate of the intersection points can be obtained. The equation of a line drawn parallel to y-axis in the horizontal 2D plane formed at lower face of TESR passing through the point $\mathrm{p}_{10}$, into the equation of the bounding ellipsoid $E_{\tau}$, the $y$-coordinate of points $\mathrm{p}^{\prime}{ }_{10}$ and $\mathrm{p}^{\prime \prime}{ }_{10}$ can be obtained. From the calculated $y$-coordinates, the minor dimensions $b_{\epsilon 2}$ and $b_{\epsilon 1}$ of the ellipses along y-axis formed at lower and face face of TESR can be obtained. The combined generalized expression for $b_{\epsilon, n}$ is expressed in simplified form as under,

$$
b_{\epsilon, n}=b_{\tau} \sqrt{\frac{a_{\tau}^{2}+b_{\tau}^{2}-2 \sigma_{n}^{2}+4 \sigma_{n} z_{o}-2 z_{o}^{2}+\left(-a_{\tau}^{2}+b_{\tau}^{2}\right) \cos \left(2 \theta_{\mathrm{los}}\right)}{a_{\tau}^{2}+b_{\tau}^{2}+\left(-a_{\tau}^{2}+b_{\tau}^{2}\right) \cos \left(2 \theta_{\mathrm{los}}\right)}} .
$$


Using the coordinates of GS's position, the horizontal deviation in the origin of ellipses can thus be found as,

$$
\Delta x_{n}=\frac{\left(a_{\tau}^{2}+b_{\tau}^{2}\right) x_{o}-\left(a_{\tau}^{2}-b_{\tau}^{2}\right) x_{o} \cos \left(2 \theta_{\mathrm{los}}\right)+\left(a_{\tau}^{2}-b_{\tau}^{2}\right)\left(\sigma_{n}-z_{o}\right) \sin \left(2 \theta_{\mathrm{los}}\right)}{a_{\tau}^{2}+b_{\tau}^{2}+\left(-a_{\tau}^{2}+b_{\tau}^{2}\right) \cos \left(2 \theta_{\mathrm{los}}\right)},
$$

where, $n=1$ represents the upper $2 \mathrm{D}$ ellipse for which $\sigma_{n}$ will be $\sigma_{c}$, and $n=2$ represents the lower 2D ellipse for which $\sigma_{n}$ will be $\sigma_{d}$. The shift of ellipsoid $E_{\tau}$ in $\mathrm{z}$ and $\mathrm{x}$ axes is shown by $x_{o}$ and $z_{o}$ (recall Fig.1) and can be calculated as,

$$
\begin{gathered}
x_{o}=\frac{d_{\mathrm{los}}}{2} \cos \theta_{\mathrm{los}} \\
z_{o}=h_{\mathrm{AS}}-\frac{d_{\mathrm{los}}}{2} \sin \theta_{\mathrm{los}}
\end{gathered}
$$

The equation of ellipsoid $E_{\tau}$ can be written as,

$$
\begin{aligned}
& \frac{\left(\left(x-x_{o}\right) \cos \left(\theta_{\mathrm{los}}\right)+\left(z-z_{o}\right) \sin \left(\theta_{\mathrm{los}}\right)\right)^{2}}{a_{\tau}^{2}}+\frac{\left(y-y_{o}\right)^{2}}{b_{\tau}^{2}} \\
& +\frac{\left(\left(z-z_{o}\right) \cos \left(\theta_{\mathrm{los}}\right)-\left(x-x_{o}\right) \sin \left(\theta_{\mathrm{los}}\right)\right)^{2}}{c_{\tau}^{2}}=1
\end{aligned}
$$

The volume of TESR can be found by integrating the area of elliptical slices in TESR over the vertical height of scattering region, which can be expressed as,

$$
V=\int_{-\sigma_{d}}^{\sigma_{c}} \pi a_{\epsilon, w} b_{\epsilon, w} \mathrm{~d} \sigma_{w}
$$

where, the parameters $a_{\epsilon, w}$ and $b_{\epsilon, w}$ represent the major and minor dimensions of a $2 \mathrm{D}$ ellipse formed at horizontal cross-section (i.e., parallel to ground plane) of virtual bounding ellipsoid $E_{\tau}$ at an arbitrary elevation $\sigma_{w}$. These dimensions can be obtained from (5) and (6) by replacing $\sigma_{n}$ with $\sigma_{w}$, i.e., $a_{\epsilon, w}=\left.a_{\epsilon, n}\right|_{\sigma_{n}=\sigma_{w}}$ and $b_{\epsilon, w}=\left.b_{\epsilon, n}\right|_{\sigma_{n}=\sigma_{w}}$. The solution for volume $V$ can be written in simplified form as,

$$
V=\frac{\sqrt{2} a_{\tau} b_{\tau}^{2} \pi\left(\sigma_{c}+\sigma_{d}\right)\left(3 a_{\tau}^{2}+3 b_{\tau}^{2}-2\left(\sigma_{c}+\sigma_{d}\right)^{2}+6\left(\sigma_{c}+\sigma_{d}\right) z_{o}-6 z_{o}^{2}+3\left(-a_{\tau}^{2}+b_{\tau}^{2}\right) \cos \left(2 \theta_{\mathrm{los}}\right)\right)}{3 \sqrt{\left(a_{\tau}^{2}+b_{\tau}^{2}+\left(-a_{\tau}^{2}+b_{\tau}^{2}\right) \cos \left(2 \theta_{\mathrm{los}}\right)\right)^{3}}}
$$

The angles in elevation and azimuth planes with a signal corresponds from a scattering point $\mathrm{S}_{\mathrm{p}}$ are represented by $\beta_{b}$ and $\phi_{b}$ at the GS and by $\beta_{a}$ and $\phi_{a}$ at the AS, respectively. Since the geometric regions of concern are below the $x-y$ plane for $z \leq 0$ when observing from either end 


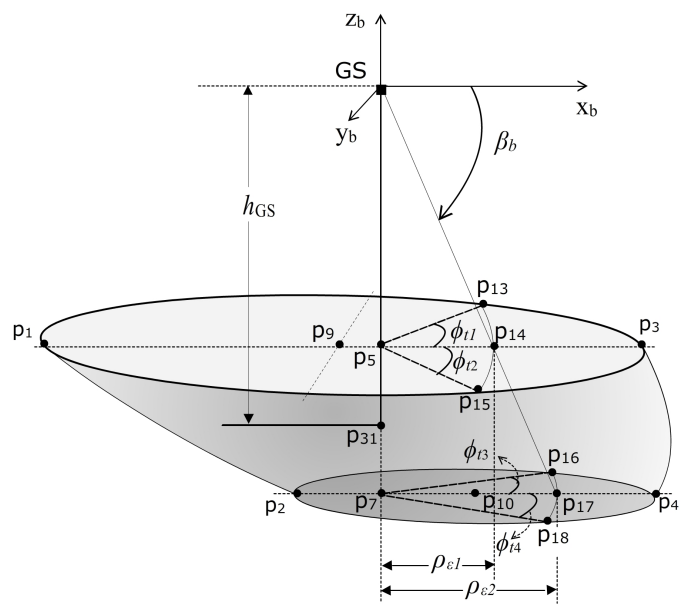

(a)

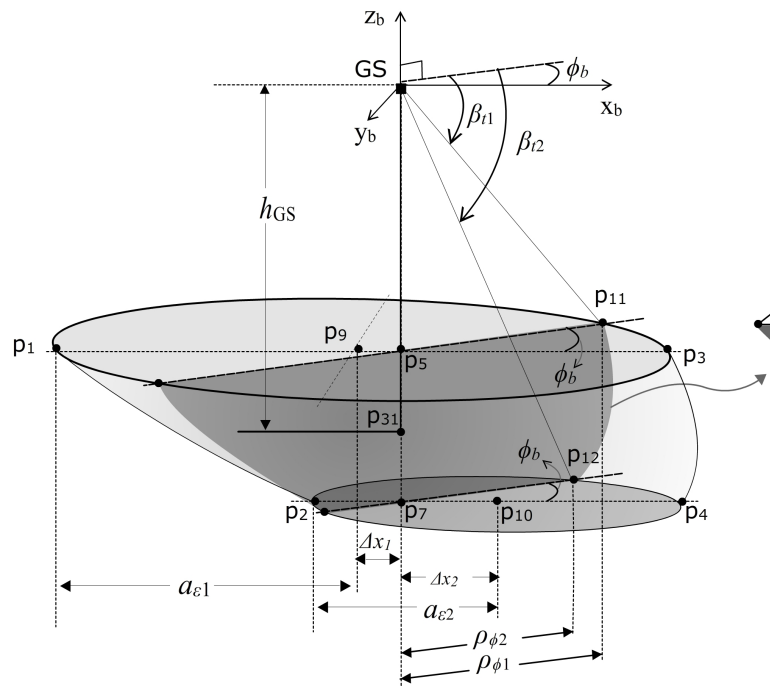

(c)

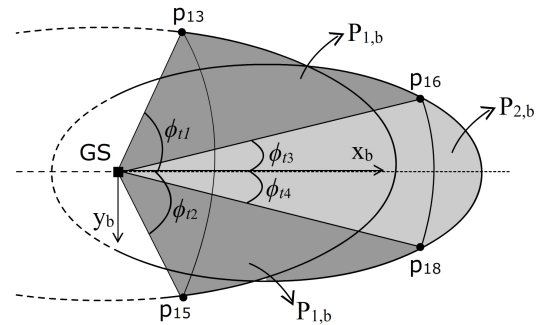

(b)

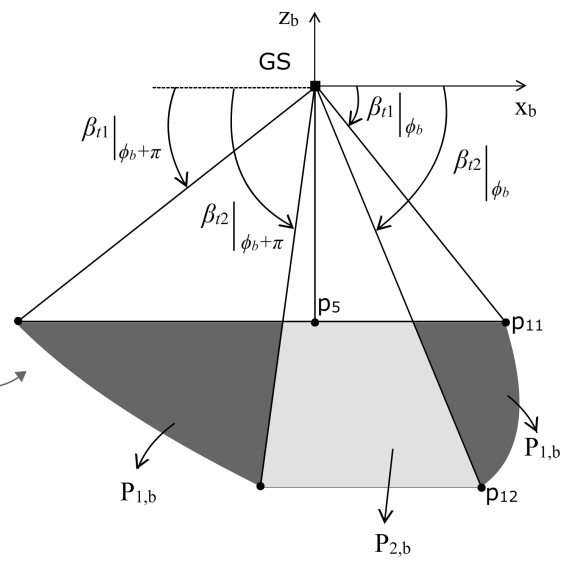

(d)

Fig. 2. Threshold angles observed from GS side of the link. (a) Azimuth threshold angles with respect to a certain elevation angle of observation, (b) Definition of angular partitions in azimuth plane, (c) Elevation threshold angles with respect to a certain azimuth angle of observation, (d) Definition of angular partitions in elevation plane.

of link; therefore, the elevation angle is defined as a positive angle in vertical plane below the $\mathrm{x}-\mathrm{y}$ plane. The distance of $\mathrm{S}_{\mathrm{p}}$ from GS and AS (via a single bounce) is $r_{b}$ and $r_{a}$, respectively. The relations for transforming the coordinates of far-end and observing communicating nodes can be expressed as,

$$
x_{b}=x_{a}+d, \quad y_{b}=y_{a}, \quad z_{b}=z_{a}-\left(h_{\mathrm{AS}}-h_{\mathrm{GS}}\right) .
$$

The view of TESR seen from GS is shown in Fig. 2. At given angle of elevation $\beta_{b}$, a circle intersects upper $2 \mathrm{D}$ ellipse with radius $\rho_{\epsilon 1}$ at points $\mathrm{p}_{13}$ and $\mathrm{p}_{15}$. Similarly, another circle intersects the lower $2 \mathrm{D}$ ellipse with radius $\rho_{\epsilon 2}$ at points $\mathrm{p}_{16}$ and $\mathrm{p}_{18}$. The angles formed in azimuth plane 
with these points can be obtained as,

$$
\left.\begin{array}{l}
\phi_{t 1} \\
\phi_{t 2}
\end{array}\right\}=\arccos \left(\frac{-b_{\epsilon 1}^{2} \Delta x_{1} \mp \sqrt{a_{\epsilon 1}^{2}\left(a_{\epsilon 1}^{2}\left(-b_{\epsilon 1}^{2}+\rho_{\epsilon 1}^{2}\right)+b_{\epsilon 1}^{2}\left(b_{\epsilon 1}^{2}-\rho_{\epsilon 1}^{2}+\Delta x_{1}^{2}\right)\right)}}{\left(a_{\epsilon 1}^{2}-b_{\epsilon 1}^{2}\right) \rho_{\epsilon 1}^{2}}\right),
$$

where, the equation for $\phi_{t 1}$ and $\phi_{t 2}$ takes the sign "-" and “+” from “F”, respectively; which is according to their corresponding vertical sequence of writing. This convention for notation of signs is followed throughout the paper.

Similarly, relationship for $\phi_{t 3}$ and $\phi_{t 4}$ may be calculated by changing the parameters of top face $2 \mathrm{D}$ ellipse (i.e., $a_{\epsilon 1}, b_{\epsilon 1}, \rho_{\epsilon 1}$, and $\left.\Delta x_{1}\right)$ with parameter of bottom face $2 \mathrm{D}$ ellipse (i.e., $a_{\epsilon 2}, b_{\epsilon 2}$, $\rho_{\epsilon 2}$, and $\left.\Delta x_{2}\right)$ in (13). Relations for $\rho_{\epsilon 1}$ and $\rho_{\epsilon 2}$ are given as,

$$
\begin{aligned}
& \rho_{\epsilon 1}=\left(h_{\mathrm{GS}}-\sigma_{c}\right) \cot \beta_{b}, \\
& \rho_{\epsilon 2}=\left(h_{\mathrm{GS}}+\sigma_{d}\right) \cot \beta_{b} .
\end{aligned}
$$

From GS, when observed at given azimuth angle $\phi_{b}$, as shown in Fig. $2, \beta_{t 1}$ and $\beta_{t 2}$ are the elevation threshold angles formed with points $\mathrm{p}_{12}$ and $\mathrm{p}_{11}$ on the lower and upper face of TESR, respectively. The mentioned angles of elevation can be obained as,

$$
\begin{aligned}
& \beta_{t 1}=\arctan \left(\frac{h_{\mathrm{GS}}-\sigma_{c}}{\rho_{\phi 1}}\right), \\
& \beta_{t 2}=\arctan \left(\frac{h_{\mathrm{GS}}+\sigma_{d}}{\rho_{\phi 2}}\right),
\end{aligned}
$$

where, $\rho_{\phi 1}$ and $\rho_{\phi 2}$ are distances in horizontal plane from GS to boundary of TESR for certain azimuth angle $\phi_{b}$. After mathematical simplifications, $\rho_{\phi 1}$ can be expressed as,

$$
\rho_{\phi 1}=\frac{-2 b_{\epsilon 1}^{2} \Delta x_{1} \cos \phi_{b}+\sqrt{2 a_{\epsilon 1}^{2} b_{\epsilon 1}^{2}\left(a_{\epsilon 1}^{2}+b_{\epsilon 1}^{2}-\Delta x_{1}^{2}+\left(-a_{\epsilon 1}^{2}+b_{\epsilon 1}^{2}+\Delta x_{1}^{2}\right) \cos 2 \phi_{b}\right)}}{a_{\epsilon 1}^{2}+b_{\epsilon 1}^{2}+\left(-a_{\epsilon 1}^{2}+b_{\epsilon 1}^{2}\right) \cos \left(2 \phi_{b}\right)} .
$$

By the same approach, relations for $\rho_{\phi 2}$ may be calculated by changing the parameters of top face 2D ellipse (i.e., $a_{\epsilon 1}, b_{\epsilon 1}$, and $\Delta x_{1}$ ) with parameters of bottom face $2 \mathrm{D}$ ellipse (i.e., $a_{\epsilon 2}, b_{\epsilon 2}$, and $\left.\Delta x_{2}\right)$ in (18).

When observing end is GS, the GS's height is usually more than the average height of rooftop (i.e., $h_{\mathrm{GS}} \geq \sigma_{c}$ ), so for simplifications in geometry, TESR can be divided in angular partitions, 
viz: $\mathrm{P}_{1, b}$ and $\mathrm{P}_{2, b}$. The definitions of these partitions based on given threshold angles observing from GS are given below,

$$
\begin{gathered}
\left\{\begin{array}{c}
\beta_{t 1} \leq \beta_{b} \leq \beta_{t 2} \\
\text { or } \\
\phi_{t 3} \leq \phi_{b} \leq \phi_{t 1} \\
\text { or } \\
\phi_{t 4} \leq \phi_{b} \leq \phi_{t 2}
\end{array}\right\} \rightarrow \mathrm{P}_{1, \mathrm{~b}}, \\
\left\{\begin{array}{c}
\beta_{t 2}<\beta_{b} \leq \frac{\pi}{2} \\
\text { or } \\
\phi_{t 4} \leq \phi_{b} \leq \phi_{t 3}
\end{array}\right\} \rightarrow \mathrm{P}_{2, \mathrm{~b}},
\end{gathered}
$$

where the symbol " $\rightarrow$ " implies to a partition name for given angular conditions. These two partitions are reflected in Fig. 2 (b) and 2 (d). When observation end is GS, the distance between nearest scatterer on surface of TESR and GS can be obtained as,

$$
r_{b, \min }= \begin{cases}\frac{h_{\mathrm{GS}}-\sigma_{c}}{\sin \beta_{b}}, & h_{\mathrm{GS}} \geq \sigma_{c} \\ 0 & , \text { otherwise. }\end{cases}
$$

The line drawn from the GS along the observing direction (i.e., at a certain azimuth angle $\phi_{b}$ and elevation angle $\beta_{b}$ ), intersects the bounding ellipsoid $E_{\tau}$ for the angular partition $\mathrm{P}_{1, \mathrm{~b}}$, and intersects the plane formed at the bottom of TESR for the angular partition $\mathrm{P}_{2, \mathrm{~b}}$. The euclidian distance from the GS to these intersection points is shown by $r_{b, \max }$, which can be expressed in simplified form for both the angular partitions as given below,

$$
r_{b, \max }=\left\{\begin{array}{cc}
\frac{1}{\left(10 a_{\tau}^{2}+6 b_{\tau}^{2}-2\left(a_{\tau}^{2}-b_{\tau}^{2}\right) \kappa_{b 5}\right)} \times\left\{2 \left(-8 a_{\tau}^{2} \kappa_{b 3}+8 b_{\tau}^{2} \kappa_{b 4}\right.\right. & \\
+\left(2 \kappa_{b 0}\left(10 a_{\tau}^{2}+6 b_{\tau}^{2}-2\left(a_{\tau}^{2}-b_{\tau}^{2}\right) \kappa_{b 5}\right)\right. & \\
\left.\left.\left.+16\left(\kappa_{b 1} \sin \beta_{b}+\kappa_{b 2} \cos \beta_{b} \cos \phi_{b}\right)^{2}\right)^{1 / 2}\right)\right\} & \text { for } \mathrm{P}_{1, \mathrm{~b}}, \\
\frac{h_{\mathrm{GS}}+\sigma_{d}}{\sin \beta_{b}} & , \text { for } \mathrm{P}_{2, \mathrm{~b}},
\end{array}\right.
$$




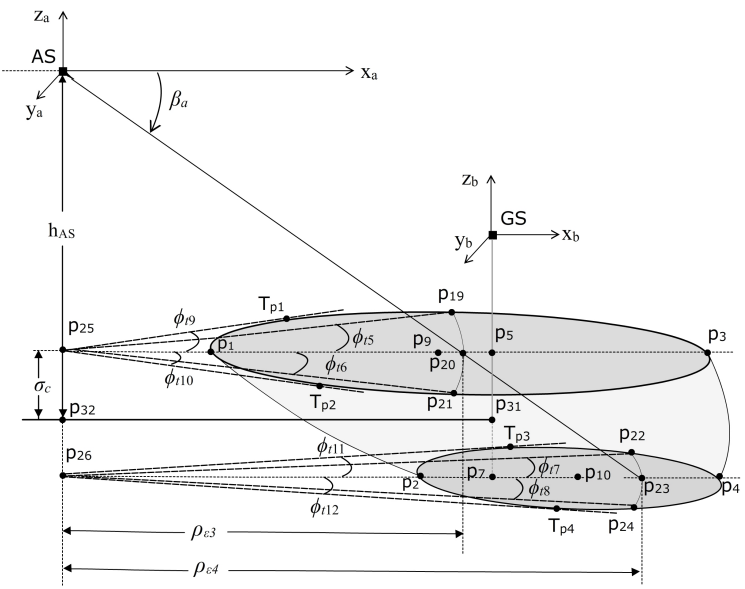

(a)

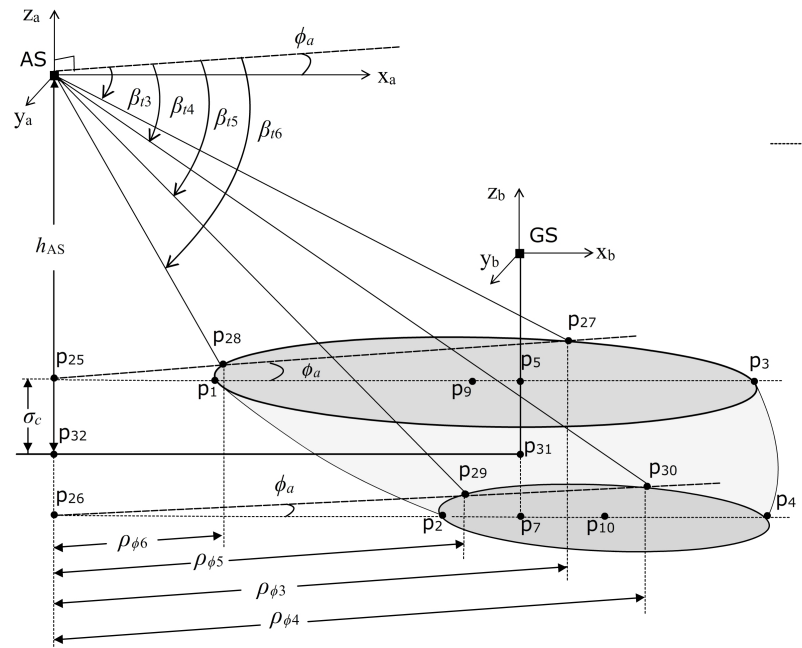

(c)

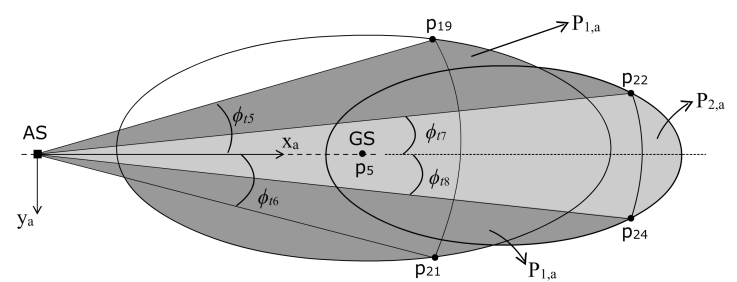

(b)

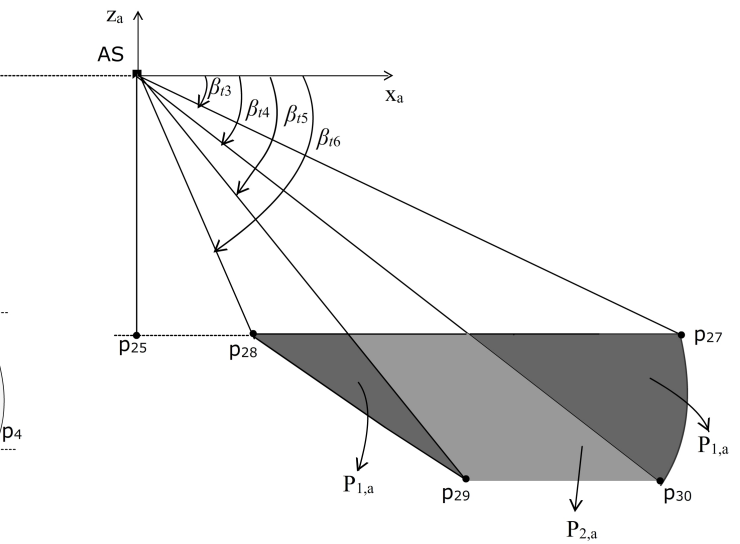

(d)

Fig. 3. Threshold angles observed from AS side of the link. (a) Azimuth threshold angles with respect to given elevation angle while observation, (b) Definition of angular partitions in azimuth plane, (c) Elevation threshold angles with respect to given azimuth angle while observation, (d) Definition of angular partitions in elevation plane.

where, the parameters $\kappa_{b 0}, \kappa_{b 1}, \kappa_{b 2}, \kappa_{b 3}, \kappa_{b 4}$, and $\kappa_{b 5}$ are given as,

$$
\begin{aligned}
& \kappa_{b 0}=a_{\tau}^{2}\left(2 b_{\tau}^{2}-x_{o}^{2}-z_{o}^{2}\right)-b_{\tau}^{2}\left(x_{o}^{2}+z_{o}^{2}\right)+\left(a_{\tau}^{2}-b_{\tau}^{2}\right)\left(\left(x_{o}^{2}-z_{o}^{2}\right)\left(\xi^{2}-\psi^{2}\right)-4 x_{o} z_{o} \xi \psi\right), \\
& \kappa_{b 1}=\left(z_{o}\left(a_{\tau}^{2}+b_{\tau}^{2}\right)+\left(a_{\tau}^{2}-b_{\tau}^{2}\right)\left(z_{o}\left(\xi^{2}-\psi^{2}\right)+2 x_{o} \xi \psi\right)\right) \\
& \kappa_{b 2}=\left(-x_{o}\left(a_{\tau}^{2}+b_{\tau}^{2}\right)+\left(a_{\tau}^{2}-b_{\tau}^{2}\right)\left(x_{o}\left(\xi^{2}-\psi^{2}\right)-2 z_{o} \xi \psi\right)\right), \\
& \kappa_{b 3}=\left(z_{o} \xi+x_{o} \psi\right)\left(\xi \sin \beta_{b}-\psi \cos \beta_{b} \cos \phi_{b}\right), \\
& \kappa_{b 4}=\left(x_{o} \xi-z_{o} \psi\right)\left(\xi \cos \beta_{b} \cos \phi_{b}+\psi \sin \beta_{b}\right), \\
& \kappa_{b 5}=-\left(\xi^{2}-\psi^{2}\right)+\cos \left(2 \beta_{b}\right)\left(-1+3\left(\xi^{2}-\psi^{2}\right)\right)+4 \xi^{2} \cos ^{2} \beta_{b} \cos \left(2 \phi_{b}\right)+8 \xi \psi \cos \phi_{b} \sin \left(2 \beta_{b}\right),
\end{aligned}
$$

and $\xi=\cos \theta_{\text {los }}$ and $\psi=\sin \theta_{\text {los }}$.

The TESR is shown in Fig. 3 when observing end is AS. For a given angle of elevation $\beta_{a}$, a 
circle intersects upper $2 \mathrm{D}$ ellipse with radius $\rho_{\epsilon 3}$ at points $\mathrm{p}_{19}$ and $\mathrm{p}_{21}$ and another circle intersects the lower $2 \mathrm{D}$ ellipse with radius $\rho_{\epsilon 4}$ at points $\mathrm{p}_{22}$ and $\mathrm{p}_{24}$. The angles formed in azimuth plane with these points are shown with $\left(\phi_{t 5}\right.$ and $\left.\phi_{t 6}\right)$ and given as,

$$
\left.\begin{array}{c}
\phi_{t 5} \\
\phi_{t 6}
\end{array}\right\}=\arccos \left(\frac{-b_{\epsilon 1}^{2} d+b_{\epsilon 1}^{2} \Delta x_{1} \mp \sqrt{a_{\epsilon 1}^{2}\left(a_{\epsilon 1}^{2}\left(-b_{\epsilon 1}^{2}+\rho_{\epsilon 3}^{2}\right)+b_{\epsilon 1}^{2}\left(b_{\epsilon 1}^{2}+d^{2}-\rho_{\epsilon 3}^{2}-2 d \Delta x_{1}+\Delta x_{1}^{2}\right)\right)}}{\left(a_{\epsilon 1}^{2}-b_{\epsilon 1}^{2}\right) \rho_{\epsilon 3}^{2}}\right) .
$$

Similarly, relationship for $\phi_{t 7}$ and $\phi_{t 8}$ can be calculated by changing the parameters of upper 2D ellipse (i.e., $a_{\epsilon 1}, b_{\epsilon 1}, \rho_{\epsilon 3}$, and $\Delta x_{1}$ ) with parameters of lower $2 \mathrm{D}$ ellipse (i.e., $a_{\epsilon 2}, b_{\epsilon 2}, \rho_{\epsilon 4}$, and $\left.\Delta x_{2}\right)$ in (29). Relations for $\rho_{\epsilon 3}$ and $\rho_{\epsilon 4}$ are given as,

$$
\begin{aligned}
& \rho_{\epsilon 3}=\left(h_{\mathrm{AS}}-\sigma_{c}\right) \cot \beta_{a}, \\
& \rho_{\epsilon 4}=\left(h_{\mathrm{AS}}+\sigma_{d}\right) \cot \beta_{a} .
\end{aligned}
$$

The maximum azimuthal angles are those angles which are formed by lines drawn horizontally to the tangent points $\mathrm{T}_{\mathrm{p} 1}$ and $\mathrm{T}_{\mathrm{p} 2}$ from $\mathrm{AS}$ on top face $2 \mathrm{D}$ ellipse, and represented by $\phi_{t 9}$ and $\phi_{t 10}$.

$$
\left.\begin{array}{l}
\phi_{t 9} \\
\phi_{t 10}
\end{array}\right\}=\mp \arctan \left(\frac{b_{\epsilon 1}}{\sqrt{-a_{\epsilon 1}^{2}+\left(d-\Delta x_{1}\right)^{2}}}\right) \text {. }
$$

In a similar way, the threshold angles in azimuth plane $\phi_{t 11}$ and $\phi_{t 12}$ formed at bottom of TESR can be calculated by changing the parameters of upper $2 \mathrm{D}$ ellipse (i.e., $a_{\epsilon 1}, b_{\epsilon 1}$, and $\Delta x_{1}$ ) with parameters of lower 2D ellipse (i.e., $a_{\epsilon 2}, b_{\epsilon 2}$, and $\Delta x_{2}$ ) of TESR in (32). At a given angle in azimuth plane $\phi_{a}$ as shown in Fig. 3, $\beta_{t 3}$ and $\beta_{t 6}$ are threshold angles in elevation plane subtended with points $\mathrm{p}_{27}$ and $\mathrm{p}_{28}$ on the $2 \mathrm{D}$ ellipse formed at upper part of TESR, and given as below,

$$
\begin{aligned}
& \beta_{t 3}=\arctan \left(\frac{h_{\mathrm{AS}}-\sigma_{c}}{\rho_{\phi 3}}\right), \\
& \beta_{t 6}=\arctan \left(\frac{h_{\mathrm{AS}}-\sigma_{c}}{\rho_{\phi 6}}\right) .
\end{aligned}
$$

Similarly, $\beta_{t 4}$ and $\beta_{t 5}$ are threshold angle in elevation plane formed points $\mathrm{p}_{29}$ and $\mathrm{p}_{30}$ on the lower 2D ellipse of TESR, and shown as below,

$$
\begin{aligned}
& \beta_{t 4}=\arctan \left(\frac{h_{\mathrm{AS}}+\sigma_{d}}{\rho_{\phi 4}}\right), \\
& \beta_{t 5}=\arctan \left(\frac{h_{\mathrm{AS}}+\sigma_{d}}{\rho_{\phi 5}}\right) .
\end{aligned}
$$

For a given angle in azimuth plane, $\phi_{a}, \rho_{\phi 3}$ and $\rho_{\phi 6}$ are distances in the horizontal plane from 
AS to points of intersection formed at the edge of top face of TESR. $\rho_{\phi 4}$ and $\rho_{\phi 5}$ are the distances in horizontal plane from AS to edge of bottom face of scattering region. By doing some simplifications mathematically, we can obtain these distances as below,

$$
\begin{aligned}
& \left.\begin{array}{l}
\rho_{\phi 3} \\
\rho_{\phi 6}
\end{array}\right\}=\left(\frac{1}{a_{\epsilon 1}^{2}+b_{\epsilon 1}^{2}+\left(-a_{\epsilon 1}^{2}+b_{\epsilon 1}^{2}\right) \cos 2 \phi_{a}} \times\left\{-2 b_{\epsilon 1}^{2}\left(d-\Delta x_{1}\right) \cos \phi_{a}\right.\right. \\
& \pm\left(2 a _ { \epsilon 1 } ^ { 2 } b _ { \epsilon 1 } ^ { 2 } \operatorname { c o s } ^ { 2 } \phi _ { a } \left(a_{\epsilon 1}^{2}+\left(b_{\epsilon 1}+2 d-\Delta x_{1}\right)\left(b_{\epsilon 1}-2 d+\Delta x_{1}\right)\right.\right. \\
& \left.\left.\left.\left.+\left(-a_{\epsilon 1}^{2}+b_{\epsilon 1}^{2}+\left(-2 d+\Delta x_{1}\right)^{2}\right) \cos \left(2 \phi_{a}\right)\right)\right)^{1 / 2} \sec \phi_{a}+2 a_{\epsilon 1}^{2} d \sin \phi_{a} \tan \phi_{a}\right\}\right) \text {. }
\end{aligned}
$$

Similarly, $\rho_{\phi 4}$ and $\rho_{\phi 5}$ can be calculated by changing the parameters of top $2 \mathrm{D}$ ellipse (i.e., $a_{\epsilon 1}$, $b_{\epsilon 1}$, and $\Delta x_{1}$ ) with parameters of bottom face 2D ellipse (i.e., $a_{\epsilon 2}, b_{\epsilon 2}$, and $\Delta x_{2}$ ) in (37). The angles $\beta_{\min }$ and $\beta_{\max }$ are limits of AoA in elevation plane when observing from AS, which can be obtained as,

$$
\begin{aligned}
& \beta_{\min }=\arctan \left(\frac{h_{\mathrm{AS}}-\sigma_{c}}{d-\Delta x_{1}+a_{\epsilon 1}}\right), \\
& \beta_{\max }=\arctan \left(\frac{h_{\mathrm{AS}}-\sigma_{c}}{d-\Delta x_{1}-a_{\epsilon 1}}\right) .
\end{aligned}
$$

For simplifications in geometry, TESR is divided into two angular partitions, and definitions of these partitions are given below based on threshold angles,

$$
\left.\begin{array}{c}
\left.\begin{array}{c}
\beta_{t 3} \leq \beta_{a} \leq \beta_{t 4} \\
\text { or } \\
\beta_{t 5} \leq \beta_{a} \leq \beta_{t 6} \\
\text { or } \\
\phi_{t 7} \leq \phi_{a} \leq \phi_{t 5} \\
\text { or } \\
\phi_{t 8} \leq \phi_{a} \leq \phi_{t 6}
\end{array}\right\} \rightarrow \mathrm{P}_{1, \mathrm{a}}, \\
\text { or } \\
\beta_{t 4}<\beta_{a} \leq \beta_{t 5} \\
\phi_{t 8} \leq \phi_{a} \leq \phi_{t 7}
\end{array}\right\} \rightarrow \mathrm{P}_{2, \mathrm{a}} .
$$

These two partitions are shown in Fig. 3 (b) and (d). When observation end is AS, the distance 
between nearest scatterer on surface of TESR and AS can be obtained as,

$$
r_{a, \min }=\frac{h_{\mathrm{AS}}-\sigma_{c}}{\sin \beta_{a}}
$$

However, the distance between farthest scatterer on surface of TESR is given below in terms of elevation and azimuth angles seen at AS (i.e., $\beta_{a}$ and $\phi_{a}$ ),

$$
r_{a, \max }=\left\{\begin{array}{cc}
\frac{1}{\left(10 a_{\tau}^{2}+6 b_{\tau}^{2}-2\left(a_{\tau}^{2}-b_{\tau}^{2}\right) \kappa_{a 5}\right)} \times\left\{2 \left(8 a_{\tau}^{2} \kappa_{a 3}+8 b_{\tau}^{2} \kappa_{a 4}\right.\right. & \\
+\left(2 \kappa_{a 0}\left(10 a_{\tau}^{2}+6 b_{\tau}^{2}-2\left(a_{\tau}^{2}-b_{\tau}^{2}\right) \kappa_{a 5}\right)\right. & \\
\left.\left.\left.\quad+16\left(\kappa_{a 1} \sin \beta_{a}-\kappa_{a 2} \cos \beta_{a} \cos \phi_{a}\right)^{2}\right)^{1 / 2}\right)\right\} & \text { for } \mathrm{P}_{1, \mathrm{a}}, \\
\frac{h_{\mathrm{AS}}+\sigma_{d}}{\sin \beta_{a}} & \text { for } \mathrm{P}_{2, \mathrm{a}},
\end{array}\right.
$$

where, the parameters $\kappa_{a 0}, \kappa_{a 1}, \kappa_{a 2}, \kappa_{a 3}, \kappa_{a 4}$, and $\kappa_{a 5}$ are given below,

$$
\begin{aligned}
& \kappa_{a 0}=a_{\tau}^{2}\left(2 b_{\tau}^{2}-x_{o}^{2}-z_{o}^{2}\right)-b_{\tau}^{2}\left(x_{o}^{2}+z_{o}^{2}\right)+\left(a_{\tau}^{2}-b_{\tau}^{2}\right)\left(\left(x_{o}^{2}-z_{o}^{2}\right)\left(\xi^{2}-\psi^{2}\right)+4 x_{o} z_{o} \xi \psi\right) \\
& \kappa_{a 1}=\left(z_{o}\left(a_{\tau}^{2}+b_{\tau}^{2}\right)+\left(a_{\tau}^{2}-b_{\tau}^{2}\right)\left(z_{o}\left(\xi^{2}-\psi^{2}\right)-2 x_{o} \xi \psi\right)\right) \\
& \kappa_{a 2}=\left(-x_{o}\left(a_{\tau}^{2}+b_{\tau}^{2}\right)+\left(a_{\tau}^{2}-b_{\tau}^{2}\right)\left(x_{o}\left(\xi^{2}-\psi^{2}\right)+2 z_{o} \xi \psi\right)\right) \\
& \kappa_{a 3}=\left(z_{o} \xi-x_{o} \psi\right)\left(\xi \sin \beta_{a}-\psi \cos \beta_{a} \cos \phi_{a}\right) \\
& \kappa_{a 4}=\left(x_{o} \xi+z_{o} \psi\right)\left(\xi \cos \beta_{a} \cos \phi_{a}+\psi \sin \beta_{a}\right) \\
& \kappa_{a 5}=-\left(\xi^{2}-\psi^{2}\right)+\cos \left(2 \beta_{a}\right)\left(-1+\left(\xi^{2}-\psi^{2}\right)\right)+4 \xi^{2} \cos ^{2} \beta_{a} \cos \left(2 \phi_{a}\right)+8 \xi \psi \cos \left(\phi_{a}\right) \sin \left(2 \beta_{a}\right)
\end{aligned}
$$

The proposed mathematical model assumed the curvature of earth as a flat surface. The percentage of the major dimension of TESR along x-axis (i.e., $2 a_{\epsilon 1}$ ) compared to the LoS distance is numerically observed to be about $3 \%-28 \%$ for the the length of longest propagation path $1.01-1.4$ times the length of LoS path. This further results in an elevational angular span (i.e., $\left.\beta_{\text {span }}=\beta_{\max }-\beta_{\min }\right)$ of $1^{o}-32^{\circ}$ for the length of longest propagation path $1.01-1.4$ times the length of LoS path. Therefore, error in accuracy of the proposed model due to the assumption of earth's flat surface is considered as tolerable for such A2G communication scenarios. The knowledge of azimuth and elevation angular spread help in determining the spatial coherence of a certain radio propagation environment. Azimuthal angular span observed at the AS can be 
obtained from the derived azimuth threshold angles (given in (32)), which is given as under,

$$
\phi_{\mathrm{span}}=\phi_{t 9}-\phi_{t 10}
$$

Similarly, relationships for elevational angular span can also be obtained from the elevation threshold angles. In a typical A2G communication environment, the available knowledge of azimuth or elevational angular spread can be used to obtained the delay of longest propagation path $\tau_{\max }$. The relationship of $\tau_{\max }$ with $\phi_{\text {span }}$ can be obtained by solving (50) for $\tau_{\max }$. The simplified solution is expressed as follows,

$$
\begin{aligned}
\tau_{\max }= & \frac{1}{2 c^{2} \sqrt{2}}\left(c^{2} \kappa_{\tau}-\cos \left(\frac{\phi_{\text {span }}}{2}\right)^{2}\left(c ^ { 4 } \operatorname { s e c } ( \frac { \phi _ { \text { span } } } { 2 } ) ^ { 2 } \left(\kappa_{\tau}^{2} \sec \left(\frac{\phi_{\text {span }}}{2}\right)^{2}-32 d_{\mathrm{los}}^{2}\right.\right.\right. \\
& \times\left(8 \sec \left(\frac{\phi_{\text {span }}}{2}\right)^{2}\left(\left(d-x_{o}\right) \cos \theta_{\mathrm{los}}+\left(\sigma_{c}-z_{o}\right) \sin \theta_{\mathrm{los}}\right)^{2}+2 \cos \theta_{\mathrm{los}}\left(\left(d_{\mathrm{los}}^{2}\right.\right.\right. \\
& \left.\left.\left.\left.\left.\left.-4\left(d-x_{o}\right)^{2}+4\left(\sigma_{c}-z_{o}\right)^{2}\right) \cos \theta_{\mathrm{los}}-8\left(d-x_{o}\right)\left(\sigma_{c}-z_{o}\right) \sin \theta_{\mathrm{los}}\right)\right)\right)\right)^{1 / 2}\right)^{1 / 2},
\end{aligned}
$$

where, the parameter $\kappa_{\tau}$, is given as,

$$
\begin{aligned}
\kappa_{\tau}=5 d_{\mathrm{los}}^{2} & +8\left(d-x_{o}\right)^{2}+16\left(\sigma_{c}-z_{o}\right)^{2}+\left(d_{\mathrm{los}}^{2}-8\left(d-x_{o}\right)^{2}\right) \cos \phi_{\mathrm{span}} \\
& +d_{\mathrm{los}}^{2} \cos \left(2 \theta_{\mathrm{los}}\right)\left(1+\cos \phi_{\mathrm{span}}\right) .
\end{aligned}
$$

III. SPATIAL CHARACTERISTICS OF THE PROPOSED MODEL

In this section, expressions for the joint PDF of AoA in elevation and azimuth planes are derived for proposed generalized 3D A2G channel model. The derivation of joint and marginal PDFs of AoA seen at AS and GS is given in Sec. III-A and Sec. III-B respectively. Results and discussions on AoA are discussed in III-C.

\section{A. PDF of AoA at AS}

The joint density function can be written as below as a function of distance $r_{a}$ and angles seen at AS,

$$
p\left(r_{a}, \phi_{a}, \beta_{a}\right)=\frac{f\left(\mathrm{x}_{\mathrm{a}}, \mathrm{y}_{\mathrm{a}}, \mathrm{z}_{\mathrm{a}}\right)}{\left|J\left(\mathrm{x}_{\mathrm{a}}, \mathrm{y}_{\mathrm{a}}, \mathrm{z}_{\mathrm{a}}\right)\right| \mid} \begin{aligned}
& \mathrm{x}_{\mathrm{a}}=r_{a} \cos \beta_{a} \cos \phi_{a} \\
& \mathrm{y}_{\mathrm{a}}=r_{a} \cos \beta_{a} \sin \phi_{a} \\
& \mathrm{z}_{\mathrm{a}}=r_{a} \sin \beta_{a}
\end{aligned}
$$

The Jacobean transformation $J\left(\mathrm{x}_{\mathrm{a}}, \mathrm{y}_{\mathrm{a}}, \mathrm{z}_{\mathrm{a}}\right)$ given in (53) can be found as,

$$
J\left(\mathrm{x}_{\mathrm{a}}, \mathrm{y}_{\mathrm{a}}, \mathrm{z}_{\mathrm{a}}\right)=\frac{1}{r_{a}^{2} \cos \beta_{a}} .
$$


When the distribution of the scatterers is assumed as uniform in the TESR with volume $V$, then the density function of scatterers may be written as,

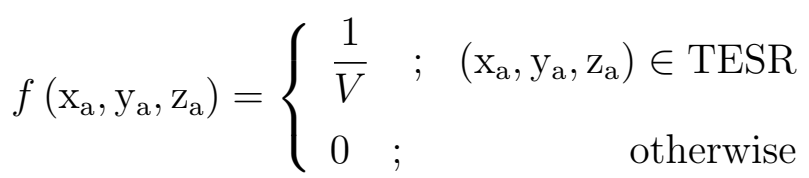

After substituting (54) and (55) in (53), the joint density function may be given as,

$$
p\left(r_{a}, \phi_{a}, \beta_{a}\right)=\frac{r_{a}^{2} \cos \beta_{a}}{V} .
$$

The joint PDF expression of AoA can be found in closed-form for elevation and azimuth angles by integration of (56) over $r_{a}$ for given limits,

$$
p\left(\phi_{a}, \beta_{a}\right)=\frac{\left(r_{a, \max }^{3}-r_{a, \min }^{3}\right) \cos \beta_{a}}{3 V} .
$$

The marginal PDF of AoA in azimuth plane can be obtained by integration of (57) over $\beta_{a}$ for given limits, and given as below,

$$
\begin{array}{r}
p\left(\phi_{a}\right)=\left.\int_{\beta_{t 4}}^{\beta_{t 5}} p\left(\phi_{a}, \beta_{a}\right)\right|_{\mathrm{P}_{2, \mathrm{a}}} \mathrm{d} \beta_{a}+\left.\int_{\beta_{t 3}}^{\beta_{t 4}} p\left(\phi_{a}, \beta_{a}\right)\right|_{\mathrm{P}_{1, \mathrm{a}}} \mathrm{d} \beta_{a}+\left.\int_{\beta_{t 5}}^{\beta_{t 6}} p\left(\phi_{a}, \beta_{a}\right)\right|_{\mathrm{P}_{1, \mathrm{a}}} \mathrm{d} \beta_{a} \\
,-\phi_{t 10} \leq \phi_{a} \leq \phi_{t 9} .
\end{array}
$$

Similarly, the expression for marginal PDF can be obtained in elevation plane by integration of (57) over $\phi_{a}$ for given limits,

$$
\begin{array}{r}
p\left(\beta_{a}\right)=\left.\int_{\phi_{t 7}}^{\phi_{t 5}} p\left(\phi_{a}, \beta_{a}\right)\right|_{\mathrm{P}_{1, \mathrm{a}}} \mathrm{d} \phi_{a}+\left.\int_{\phi_{t 8}}^{\phi_{t 6}} p\left(\phi_{a}, \beta_{a}\right)\right|_{\mathrm{P}_{1, \mathrm{a}}} \mathrm{d} \phi_{a}+\left.\int_{\phi_{t 8}}^{\phi_{t 7}} p\left(\phi_{a}, \beta_{a}\right)\right|_{\mathrm{P}_{2, \mathrm{a}}} \mathrm{d} \phi_{a} \\
, \beta_{\min } \leq \beta_{a} \leq \beta_{\max } .
\end{array}
$$

The marginal PDF can be found by numerically solving the integrations given in (58) and (59).

\section{B. PDF of AoA at GS}

PDFs of AoA observed at GS for the proposed 3D model are derived in this section. The joint function corresponded with the angles observed at GS and the distance $r_{b}$ is given below with similar method as in (53),

$$
p\left(r_{b}, \phi_{b}, \beta_{b}\right)=\frac{r_{b}^{2} \cos \beta_{b}}{V}
$$


The joint PDF expression of AoA can be found in closed-form for elevation and azimuth angles by integration of (60) over $r_{b}$ for given limits,

$$
p\left(\phi_{b}, \beta_{b}\right)=\frac{\left(r_{b, \max }^{3}-r_{b, \min }^{3}\right) \cos \beta_{b}}{3 V} .
$$

The marginal PDF of AoA in azimuth plane can be obtained by integration of (61) over $\beta_{b}$ for given limits, and shown as,

$$
\begin{aligned}
p\left(\phi_{b}\right)=\left.\int_{\beta_{t 1}}^{\beta_{t 2}} p\left(\phi_{b}, \beta_{b}\right)\right|_{\mathrm{P}_{1, \mathrm{~b}}} \mathrm{~d} \beta_{b}+\left.\int_{\beta_{t 2}}^{\frac{\pi}{2}} p\left(\phi_{b}, \beta_{b}\right)\right|_{\mathrm{P}_{2, \mathrm{~b}}} \mathrm{~d} \beta_{b} \\
\quad,-\pi \leq \phi_{b} \leq \pi .
\end{aligned}
$$

In a similar way, expression for marginal PDF can be obtained in elevation plane by integration of(61) over $\phi_{b}$ for given limits,

$$
\begin{array}{r}
p\left(\beta_{b}\right)=\left.\int_{\phi_{t 3}}^{\phi_{t 1}} p\left(\phi_{b}, \beta_{b}\right)\right|_{\mathrm{P}_{1, \mathrm{~b}}} \mathrm{~d} \phi_{b}+\left.\int_{\phi_{t 4}}^{\phi_{t 2}} p\left(\phi_{b}, \beta_{b}\right)\right|_{\mathrm{P}_{1, \mathrm{~b}}} \mathrm{~d} \phi_{b}+\left.\int_{\phi_{t 4}}^{\phi_{t 3}} p\left(\phi_{b}, \beta_{b}\right)\right|_{\mathrm{P}_{2, \mathrm{~b}}} \mathrm{~d} \phi_{b} \\
, \frac{-\pi}{2} \leq \beta_{b} \leq \frac{\pi}{2} .
\end{array}
$$

The solution for these marginal PDFs can be found by techniques of numerical integration.

\section{Results and Discussion on AoA}

In this section, a detailed discussion on the results for AoA is presented. The impact of the changing the AS's height $\left(h_{\mathrm{AS}}\right)$ on marginal PDF of AoA seen from AS is plotted in Fig. 4. The azimuthal angular distribution of energy (see Fig. 4 (a)) in the angles close to LoS direction increases with increasing $h_{\mathrm{AS}}$. Furthermore, it can bee seen in Fig. 4 (b) angular deviation of elevation AoA increases with decrease in $h_{\mathrm{AS}}$, with mean of its distribution at LoS elevation angle. Thus, it is established that in A2G scenarios when AS is located at low elevation, scatterers in elevation plane need to be considered for when modeling the effective region of scatterers around GS.

The joint PDF of AoA seen from GS is shown in Fig. 5 for different values of $\sigma_{d}$. The plot in Fig.5 (a) shows A2G communication when GS is on the ground surface (i.e., $\sigma_{d}$ taken as $0 \mathrm{~m}$ ) and the plot in Fig.5 (b) shows A2G communication when the GS is over sea surface (i.e., $\sigma_{d}$ taken as $35 \mathrm{~m})$. The angular spread along elevation axis can be observed as increasing with an increase 


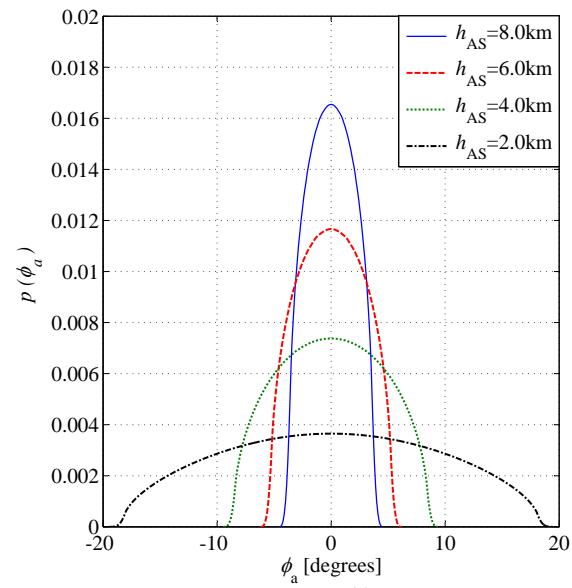

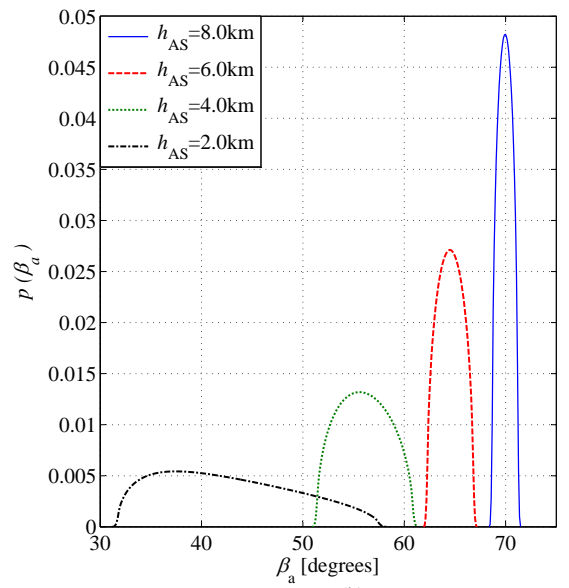

(b)

Fig. 4. The effect of $h_{\mathrm{AS}}$ on marginal PDF of AoA observed from AS (a) in azimuth plane, (b) in elevation plane, $\left(h_{\mathrm{GS}}=30 \mathrm{~m}\right.$, $\sigma_{t}=20 \mathrm{~m}, \psi_{w}=5 \mathrm{~m}, \tau_{\max }=32.1 \mu \mathrm{sec}, d=3 \mathrm{~km}$, and $\left.\sigma_{c}=20 \mathrm{~m}\right)$.
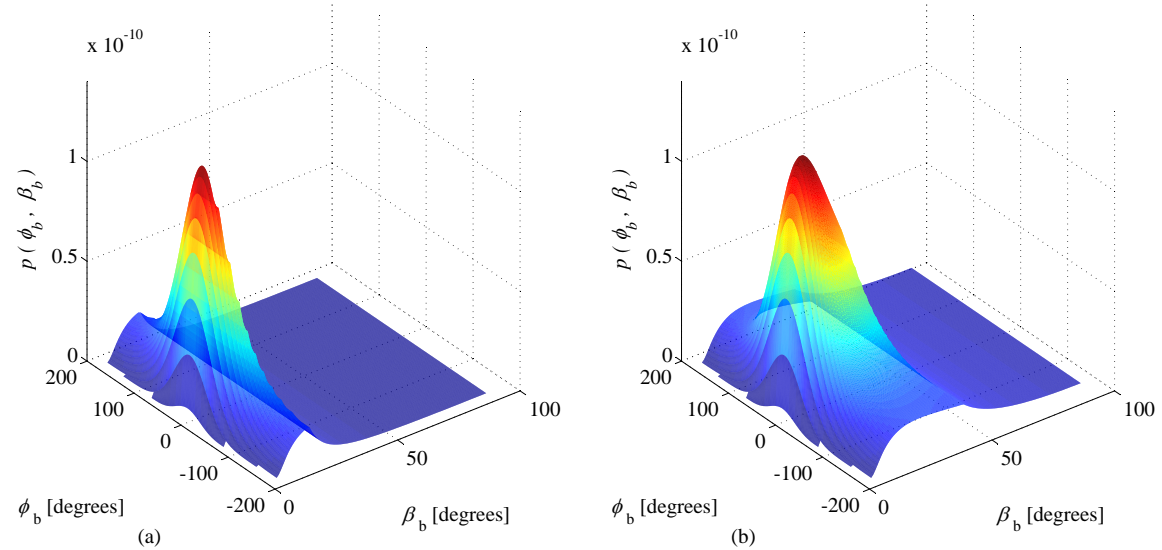

Fig. 5. Joint PDF of AoA observed from GS (a) A2G scenario when GS is on ground surface, i.e., $\sigma_{t}=0 \mathrm{~m}$ and $\psi_{w}=0 \mathrm{~m}$, (b) A2G scenario when GS is on sea surface, i.e., $\sigma_{t}=30 \mathrm{~m}$ and $\psi_{w}=5 \mathrm{~m},\left(h_{\mathrm{AS}}=9 \mathrm{~km}, h_{\mathrm{GS}}=40 \mathrm{~m}, d=3 \mathrm{~km}, \tau_{\mathrm{max}}=32.2 \mu \mathrm{sec}\right.$, and $\left.\sigma_{c}=30 \mathrm{~m}\right)$.

in $\sigma_{d}$. This also establishes the importance of inclusion of elevation axis for modeling the GS's vicinity.

In Fig. 6 (a) and (b), the effect of changint the GS's height $\left(h_{\mathrm{GS}}\right)$ on marginal PDF of AoA in azimuth and elevation planes seen at GS is illustrated. The elevation of GS has a significant impact on the mean and standard deviation of the PDF of elevation AoA; however, it has a minor impact on the distribution of energy in azimuth plane. The result is showing that it is important to increase the height of GS more as compared to the average height of rooftop level in the surroundings. The impact of vertical dimension of TESR on the PDF of AoA observed at the GS is shown in Fig. 7. It is clearly evident that increase in vertical dimensions of TESR has a significant impact 


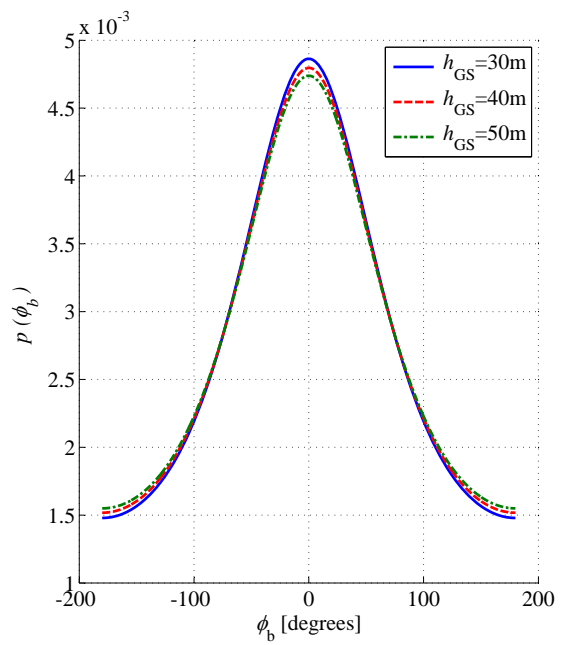

(a)

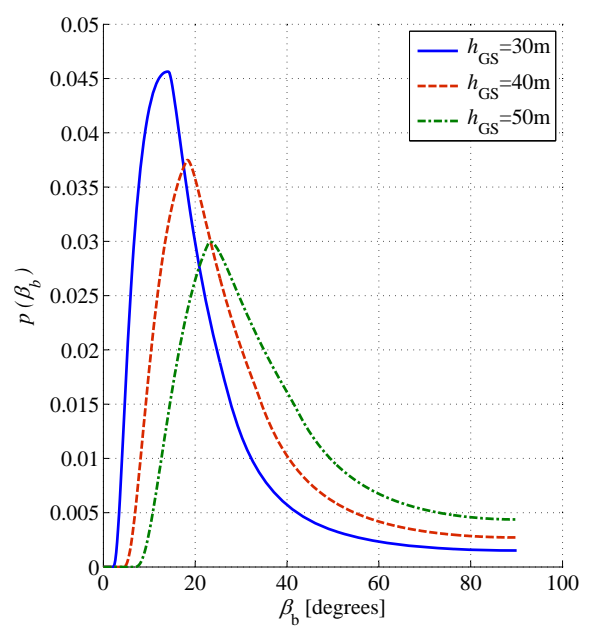

(b)

Fig. 6. The effect of $h_{\mathrm{GS}}$ on marginal PDF of AoA observed from GS (a) in azimuth plane, (b) in elevation plane, $\left(h_{\mathrm{AS}}=9 \mathrm{~km}\right.$, $\tau_{\max }=32.1 \mu \mathrm{sec}, d=3 \mathrm{~km}, \sigma_{d}=25 \mathrm{~m}$, and $\sigma_{c}=20 \mathrm{~m}$ ).
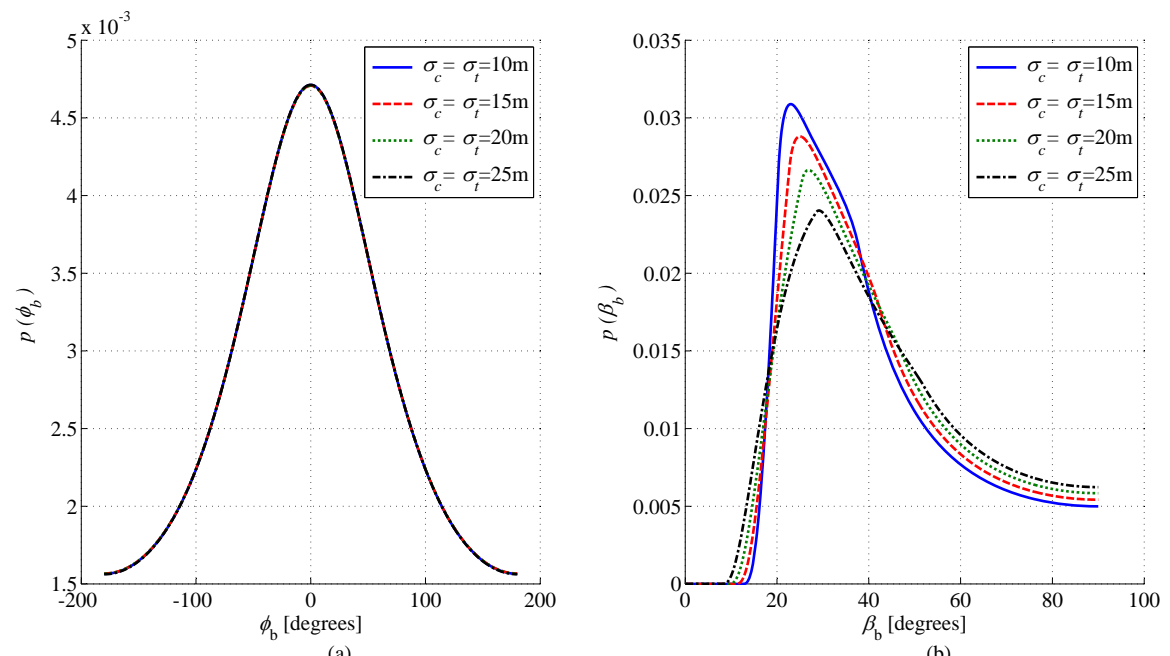

(b)

Fig. 7. The effect of $\sigma_{c}=\sigma_{t}$ on marginal PDF of AoA observed from GS (a) in azimuth plane, (b) in elevation plane, $\left(h_{\mathrm{A} S}=\right.$ $9 \mathrm{~km}, h_{\mathrm{G} S}=30 \mathrm{~m}, d=3 \mathrm{~km}$, and $\left.\tau_{\max }=32.2 \mu \mathrm{sec}\right)$.

on the PDF of elevation AoA; however, it has very minor impact on the PDF of azimuth AoA. This is because, scaling the vertical dimensions of TESR has a very minor impact on the scale along horizontal dimension. Increasing the vertical dimension of TESR changes the volume $V$, the impact due to change in the volume of TESR is not evident because of the normalization operation performed at the PDFs.

The simulations are performed for validating the analytical results by comparing with simulations results. The analytical results are also compared with results of a notable model found 

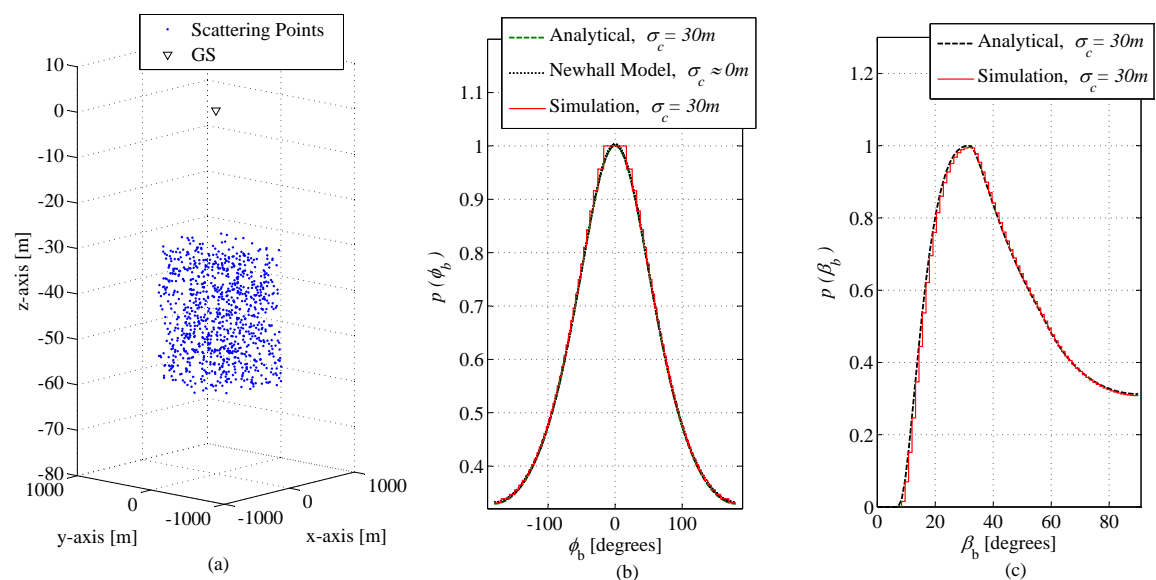

Fig. 8. (a) Distribution of scatterers used in simulation, (b) analytical and simulation results for marginal PDF of azimuth AoA seen at GS and comparison with Newhall Model, (c) analytical and simulation results for marginal PDF of elevation AoA seen at $\mathrm{GS},\left(h_{\mathrm{A} S}=9 \mathrm{~km}, h_{\mathrm{G} S}=60 \mathrm{~m}, \tau_{\max }=32.1 \mu \mathrm{sec}, \sigma_{t}=0 \mathrm{~m}, \psi_{w}=0 \mathrm{~m}, d=3 \mathrm{~km}\right.$ and Monte carlo runs $\left.=10^{4}\right)$.

in the literature. The computer simulations are done by the same approach as adopted by [37]. The scattering objects in the defined TESR are generated and shown in Fig. 8 (a). The results of PDF of AoA in azimuth plane are obtained by simulations and compared with analytical results of the proposed model and the results given for the model in [32] as shown in Fig. 8 (b). The results obtained analytically are compared with the simulations for PDF of elevation AoA in Fig. 8 (c). For $10^{3}$ scatterers distributed uniformly and results are averaged over $10^{4}$ monte carlo runs, a good match is seen between analytical and simulation results. Moreover, the proposed PDF of azimuth AoA is seen fit the results proposed by Newhall in [32] by reducing the 3D scattering region into a 2D scattering region. The model in [32] can be deduced from the proposed model by substituting a small value for the vertical dimensions of TESR, i.e., $\left(\sigma_{c}=\sigma_{t}\right) \approx 0$.

\section{TOA STATISTICS FOR THE PROPOSED MODEL}

The distribution of multipath components with respect to their delay depends on the geometric configuration of the illuminated scattering objects. The delay characteristics of the channel are important in determining the coherence bandwidth which further determines the frequency selective behavior of the channel. This section thus presents the characteristics of ToA for proposed model.

\section{A. Derivation of PDF of ToA}

In this section, the expressions are derived for joint and marginal PDF of ToA. Assume, $\tau$ is the delay of a signal reflected from a scatterer, located in TESR, may be written in terms of the 
velocity of radio wave and distance of the propagation path, as,

$$
\tau=\frac{r_{a}+r_{b}}{c} \text {. }
$$

The delay for LoS path is $\tau_{o}$, which can be expressed as,

$$
\tau_{o}=\frac{d_{\mathrm{los}}}{c}
$$

The longest path delay, $\tau_{\max }$, is assumed as known to model the dimensions of effective scattering region. This parameter can also be calculated by exploiting its geometric relationship with azimuth or elevation angular span, as presented in (51). Moreover, this parameter can also be computed by adopting reverse approach and calculating $\tau_{\max }$ from the dimensions of TESR, as provided below,

$$
\tau_{\max }=\sqrt{\left(h_{\mathrm{AS}}+\sigma_{d}\right)^{2}+\left(d+a_{\epsilon 2}-\Delta x_{2}\right)^{2}}+\sqrt{\left(h_{\mathrm{GS}}+\sigma_{d}\right)^{2}+\left(a_{\epsilon 2}-\Delta x_{2}\right)^{2}} .
$$

The delay of propagation path from certain point in TESR for given elavation and azimuth angle is shown by $\tau_{\text {lim }}$, and given below as,

$$
\tau_{\lim }\left(\phi_{b}, \beta_{b}\right)=\frac{r_{a}\left(\phi_{b}, \beta_{b}\right)+r_{b}\left(\phi_{b}, \beta_{b}\right)}{c}
$$

The distances $r_{a}$ and $r_{b}$ are distances from $\mathrm{S}_{\mathrm{p}}$ to AS and GS respectively, as shown in Fig. 1, $r_{a}$ is given in simplified form as,

$$
r_{a}\left(r_{b}, \phi_{b}, \beta_{b}\right)=\sqrt{d^{2}+r_{b}^{2} \cos ^{2} \beta_{b}-2 r_{b} d \cos \beta_{b} \cos \phi_{b}+\left(h_{\mathrm{AS}}-h_{\mathrm{GS}}+r_{b} \sin ^{2} \beta_{b}\right)} .
$$

Substituting (68) in (64) and solving for $r_{b}$, the equation can be re-arranged as,

$$
r_{b}\left(\tau, \phi_{b}, \beta_{b}\right)=\frac{d^{2}+\left(h_{\mathrm{AS}}-h_{\mathrm{GS}}\right)^{2}-c^{2} \tau^{2}}{2\left(-c \tau+d \cos \beta_{b} \cos \phi_{b}+\left(-h_{\mathrm{AS}}+h_{\mathrm{GS}}\right) \sin \beta_{b}\right)} .
$$

The boundary scatterers cause the maximum propagation path delay for a given elevation and azimuth angle. The joint function for AoA/ToA may be written [7] as,

$$
p\left(\tau, \phi_{b}, \beta_{b}\right)=\frac{p\left(r_{b}, \phi_{b}, \beta_{b}\right)}{\left|J\left(r_{b}, \phi_{b}, \beta_{b}\right)\right|} .
$$

Where $r_{b}$ is given in 69 , the Jacobean transformation $J\left(r_{b}, \phi_{b}, \beta_{b}\right)$ shown in $(70)$, can be found as,

$$
J\left(r_{b}, \phi_{b}, \beta_{b}\right)=\left|\frac{\partial r_{b}}{\partial \tau}\right|^{-1}
$$


$J\left(r_{b}, \phi_{b}, \beta_{b}\right)=\frac{2\left(c \tau-d \cos \beta_{b} \cos \phi_{b}+\left(h_{\mathrm{AS}}-h_{\mathrm{GS}}\right) \sin \beta_{b}\right)^{2}}{c\left(d^{2}+h_{\mathrm{AS}}^{2}-2 h_{\mathrm{AS}} h_{\mathrm{GS}}+h_{\mathrm{GS}}^{2}+c^{2} \tau^{2}-2 c \tau\left(d \cos \beta_{b} \cos \phi_{b}-\left(h_{\mathrm{AS}}-h_{\mathrm{GS}}\right) \sin \beta_{b}\right)\right)}$.

Substituting (72) and (60) in (70), the joint density for ToA and AoA may be expressed as,

$$
\begin{aligned}
p\left(\tau, \phi_{b}, \beta_{b}\right)= & \frac{1}{8 V\left(c \tau-d \cos \beta_{b} \cos \phi_{b}+\left(h_{\mathrm{AS}}-h_{\mathrm{GS}}\right) \sin \beta_{b}\right)^{4}} \times\left\{c \left(d^{2}+h_{\mathrm{AS}}^{2}-2 h_{\mathrm{AS}} h_{\mathrm{GS}}+\right.\right. \\
& \left.h_{\mathrm{GS}}^{2}-c^{2} \tau^{2}\right)^{2} \cos \beta_{b}\left(d^{2}+h_{\mathrm{AS}}^{2}-2 h_{\mathrm{AS}} h_{\mathrm{GS}}+h_{\mathrm{GS}}^{2}+c^{2} \tau^{2}-2 c \tau\left(d \cos \beta_{b} \cos \phi_{b}-\right.\right. \\
& \left.\left.\left.\left(h_{\mathrm{AS}}-h_{\mathrm{GS}}\right) \sin \beta_{b}\right)\right)\right\} .
\end{aligned}
$$

The joint PDF of ToA observed at GS in azimuth plane may be then calculated by integrating (73) over azimuth angle.

$$
p\left(\tau, \beta_{b}\right)=\int_{-\pi}^{\pi} p\left(\tau, \phi_{b}, \beta_{b}\right) \mathrm{d} \phi_{b} \quad, \tau_{0}<\tau \leq \tau_{\lim } \quad \text { and } \quad \frac{-\pi}{2} \leq \beta_{b} \leq \frac{\pi}{2} .
$$

Using tedious mathematical simplifications the closed form expression for $p\left(\tau, \beta_{b}\right)$ can be obtained as,

$$
\begin{aligned}
p\left(\tau, \beta_{b}\right)= & \frac{1}{\left(32 V\left(k_{3}+c \tau-d \cos \beta_{b}\right)^{4}\left(k_{3}+c \tau+d \cos \beta_{b}\right)^{3}\right)} \times\left\{k_{1} \pi\right. \\
& \times \sqrt{\frac{k_{3}+c \tau-d \cos \beta_{b}}{k_{3}+c \tau+d \cos \beta_{b}}}\left(6 d^{2} k_{2} k_{3}+8 k_{2} k_{3}^{3}-3 c \tau d^{4}+6 d^{2} k_{2} c \tau-\right. \\
& 4 d^{2} k_{3}^{2} c \tau+24 k_{2} k_{3}^{2} c \tau+16 k_{3}^{4} c \tau-20 d^{2} k_{3} c^{2} \tau^{2}+24 k_{2} k_{3} c^{2} \tau^{2}+ \\
& 48 c^{2} \tau^{2} k_{3}^{3}-16 d^{2} c^{3} \tau^{3}+8 c^{3} \tau^{3} k_{2}+48 c^{3} \tau^{3} k_{3}^{2}+16 c^{4} \tau^{4} k_{3}-2 d^{2} \times \\
& \left(-3 k_{2}\left(k_{3}+c \tau\right)+2 c \tau\left(d^{2}+k_{3}^{2}+5 c \tau k_{3}+4 c^{2} \tau^{2}\right)\right) \cos \left(2 \beta_{b}\right)-c \tau d^{4} \\
& \left.\left.\cos \left(4 \beta_{b}\right)\right)\right\},
\end{aligned}
$$

where $k_{1}, k_{2}$, and $k_{3}$ are given below,

$$
\begin{gathered}
k_{1}=c\left(d^{2}+h_{\mathrm{AS}}^{2}-2 h_{\mathrm{AS}} h_{\mathrm{GS}}+h_{\mathrm{GS}}^{2}-c^{2} \tau^{2}\right)^{2} \cos \beta_{b}, \\
k_{2}=d^{2}+h_{\mathrm{AS}}^{2}-2 h_{\mathrm{AS}} h_{\mathrm{GS}}+h_{\mathrm{GS}}^{2}+c^{2} \tau^{2}, \\
k_{3}=\left(h_{\mathrm{AS}}-h_{\mathrm{GS}}\right) \sin \beta_{b} .
\end{gathered}
$$


The joint PDF of ToA observed at GS in elevation plane can also be obtained by integrating (73) over elevation angle.

$$
p\left(\tau, \phi_{b}\right)=\int_{\frac{-\pi}{2}}^{\frac{\pi}{2}} p\left(\tau, \phi_{b}, \beta_{b}\right) \mathrm{d} \beta_{b} \quad, \tau_{0}<\tau \leq \tau_{\lim } \quad \text { and } \quad-\pi<\phi_{b} \leq \pi .
$$

Using tedious mathematical simplifications, the closed-form expression for $p\left(\tau, \phi_{b}\right)$ can be obtained as,

$$
\begin{aligned}
p\left(\tau, \phi_{b}\right)= & \frac{1}{\left(12 V\left(d^{2}+2 m_{5}+d^{2} \cos 2 \phi_{b}\right)^{3}\right)} \times\left\{c\left(d^{2}+m_{5}\right)^{2}\right. \\
& \times\left(3-\frac{2 m_{2} m_{3}^{2}\left(d^{2}+m_{5}\right)}{m_{5}^{2}}+\frac{1}{\sqrt{m_{3}}}\left\{6 \sqrt { 2 } d \left(\tanh ^{-1}\left(\sqrt{\frac{2}{m_{3}}}\left(m_{4}+h_{\mathrm{AS}}-h_{\mathrm{GS}}\right)\right)+\right.\right.\right. \\
& \left.\tanh ^{-1}\left(\sqrt{\frac{2}{m_{3}}}\left(m_{4}-h_{\mathrm{AS}}+h_{\mathrm{GS}}\right)\right)\right) \cos \phi_{b}\left(d^{4}+3 d^{2} m_{1}+2 m_{1}^{2}+c^{2} \tau^{2}\left(d^{2}-6 m_{1}\right)\right. \\
& \left.\left.+4 c^{4} \tau^{4}+d^{2}\left(d^{2}+m_{1}-7 c^{2} \tau^{2}\right) \cos \left(2 \phi_{b}\right)\right)\right\}+\left(\frac{1}{2 m_{5}^{2}} \times\left\{9 d^{6} m_{2}+64 c^{2} m_{5}^{3} \tau^{2}\right.\right. \\
& +12 d^{2} m_{5}^{2}\left(m_{1}+4 c^{2} \tau^{2}\right)+d^{4} m_{5}\left(21 m_{1}+47 c^{2} \tau^{2}\right)+4 d^{2}\left(3 m_{1}\left(d^{2}+m_{1}\right)^{2}+\right. \\
& \left.c^{2} \tau^{2}\left(3 d^{4}+5 d^{2} m_{1}+14 m_{1}^{2}\right)-c^{4} \tau^{4}\left(11 d^{2}+37 m_{1}\right)+20 c^{6} \tau^{6}\right) \\
& \left.\left.\left.\left.\cos \left(2 \phi_{b}\right)+3 d^{4}\left(d^{2} m_{2}+m_{5}^{2}\right) \cos \left(4 \phi_{b}\right)\right\}\right)\right)\right\}
\end{aligned}
$$

where, $m_{1}, m_{2}, m_{3}, m_{4}$, and $m_{5}$ are given below,

$$
\begin{gathered}
m_{1}=\left(h_{\mathrm{AS}}-h_{\mathrm{GS}}\right)^{2}, \\
m_{2}=m_{1}+c^{2} \tau^{2}, \\
m_{3}=d^{2}+2 m_{1}-2 c^{2} \tau^{2}+d^{2} \cos \phi_{b}, \\
m_{4}=c \tau+d \cos \phi_{b}, \\
m_{5}=m_{1}-c^{2} \tau^{2} .
\end{gathered}
$$


Similarly, with the angles seen at AS, the density function may be expressed as,

$$
\begin{aligned}
p\left(\tau, \phi_{a}, \beta_{a}\right) & =\frac{1}{8 V\left(c \tau-d \cos \beta_{a} \cos \phi_{a}+\left(-h_{\mathrm{AS}}+h_{\mathrm{GS}}\right) \sin \beta_{a}\right)^{4}} \times\left\{c \left(d^{2}+h_{\mathrm{AS}}^{2}-2 h_{\mathrm{AS}} h_{\mathrm{GS}}+\right.\right. \\
& \left.h_{\mathrm{GS}}^{2}-c^{2} \tau^{2}\right)^{2} \cos \beta_{a}\left(d^{2}+h_{\mathrm{AS}}^{2}-2 h_{\mathrm{AS}} h_{\mathrm{GS}}+h_{\mathrm{GS}}^{2}+c^{2} \tau^{2}-2 c \tau\left(d \cos \beta_{a} \cos \phi_{a}+\right.\right. \\
& \left.\left.\left.\left(h_{\mathrm{AS}}-h_{\mathrm{GS}}\right) \sin \beta_{a}\right)\right)\right\} .
\end{aligned}
$$

The joint PDF of ToA observed at AS in elevation and azimuth planes can thus be then obtained by integrating (86) over elevation and azimuth angles for their appropriate limits, respectively. The joint PDF of ToA may be expressed as,

$$
p\left(\tau, \beta_{a}\right)=\int_{-\phi_{\mathrm{t} 10}}^{\phi_{\mathrm{t} 9}} p\left(\tau, \phi_{a}, \beta_{a}\right) \mathrm{d} \phi_{a} \quad, \tau_{0}<\tau \leq \tau_{\lim } \quad \text { and } \quad \beta_{\min } \leq \beta_{a} \leq \beta_{\max } .
$$

The joint PDF of ToA and azimuth AoA may be expressed as,

$$
p\left(\tau, \phi_{a}\right)=\int_{\beta_{\min }}^{\beta_{\max }} p\left(\tau, \phi_{a}, \beta_{a}\right) \mathrm{d} \beta_{a} \quad, \tau_{0}<\tau \leq \tau_{\lim } \quad \text { and } \quad-\phi_{t 10} \leq \phi_{b} \leq \phi_{t 9} .
$$

The closed form expressions for (87), and (88) can also be obtained as found in (75).

The marginal PDF for ToA may be obtained by integration of any equation from (74), (79), (87), and (88) over corresponding angles, as,

$$
p(\tau)=\int_{-\pi}^{\pi} \int_{\frac{-\pi}{2}}^{\frac{\pi}{2}} p\left(\tau, \phi_{b}, \beta_{b}\right) \mathrm{d} \beta_{b} \mathrm{~d} \phi_{b} \quad, \quad \tau_{0}<\tau \leq \tau_{\max } .
$$

The closed-form expressions for $p(\tau)$ can also be obtained by using tedious mathematical simplifications. The RMS-DS, $\left(\tau_{\text {rms }}\right)$, is the square root of the second central moment of the pdf of ToA, $p(\tau)$,

$$
\tau_{\mathrm{rms}}=\sqrt{\int\left(\tau-\tau_{o}-\bar{\tau}\right)^{2} p(\tau) \mathrm{d} \tau}
$$

where, $\bar{\tau}$ is the mean excess delay which is the first moment of pdf of $\operatorname{ToA}, p(\tau)$, with respect to delay in $\operatorname{LoS}, \tau_{o}$. The expression for $\bar{\tau}$ is given below,

$$
\bar{\tau}=\int\left(\tau-\tau_{o}\right) p(\tau) \mathrm{d} \tau
$$



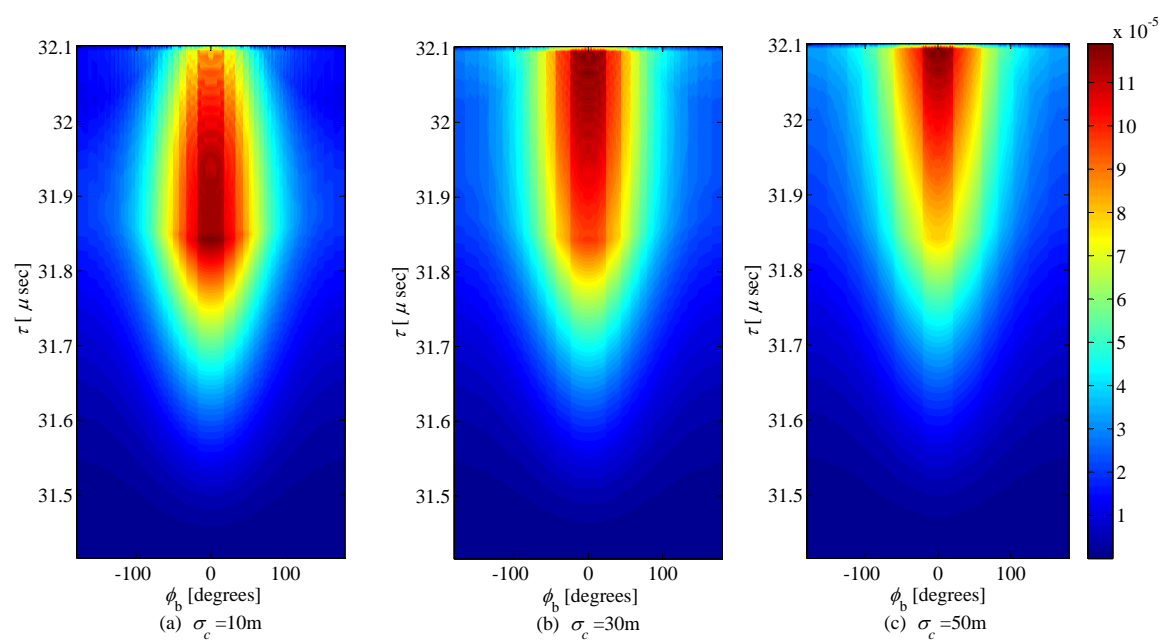

Fig. 9. Joint PDF of ToA and azimuth AoA observed when GS is at the ground surface, $\quad\left(h_{\mathrm{AS}}=9 \mathrm{~km}, h_{\mathrm{GS}}=30 \mathrm{~m}, d=3 \mathrm{~km}\right.$, $\sigma_{t}=0 \mathrm{~m}$, and $\left.\psi_{w}=0 \mathrm{~m}\right)$.
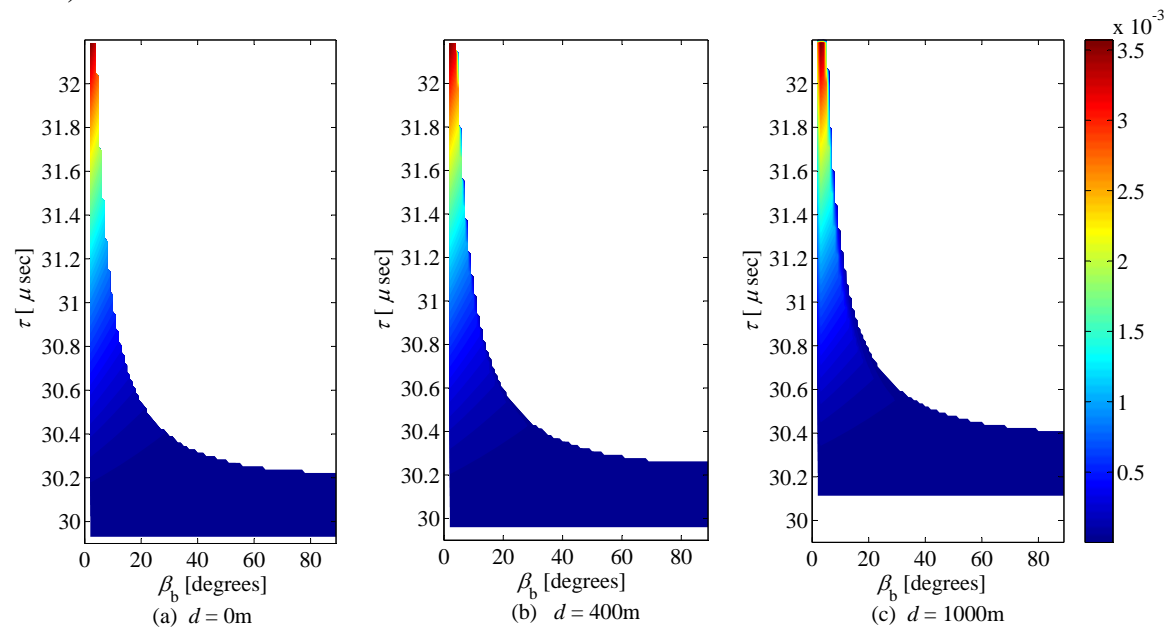

Fig. 10. Joint PDF of ToA and elevation AoA observed when GS is at the sea surface, $\left(h_{\mathrm{AS}}=9 \mathrm{~km}, h_{\mathrm{GS}}=30 \mathrm{~m}, \sigma_{t}=15 \mathrm{~m}\right.$, $\psi_{w}=5 \mathrm{~m}$, and $\left.\sigma_{c}=15 \mathrm{~m}\right)$.

\section{B. Results and Discussions on ToA}

The joint PDF for ToA w.r.t to azimuth AoA seen from the GS is shown (contour) in Fig. 9 for different channel parameters shown in caption of the plot. This plot is shown for the scenario when the GS is located at the ground surface (i.e., $\sigma_{d}=0 \mathrm{~m}$ ). In $\mathrm{A} 2 \mathrm{G}$ radio communication environment, to show the effects of average rooftop level, joint PDF of azimuth AoA and ToA is plotted in Fig. 9 (a), (b), and (c) for $\sigma_{c}$ taken as $10 \mathrm{~m}, 30 \mathrm{~m}$, and 50m, respectively. The joint PDF of elevation AoA and ToA seen from GS is shown (contour) in Fig. 10. This plot is taken for A2G communication with GS considered over sea surface (i.e., $\sigma_{d}=20 \mathrm{~m}$ ). The effect of distance between AS and GS (i.e., $d$ ) on the limits of propagation path's delay can easily be observed in 

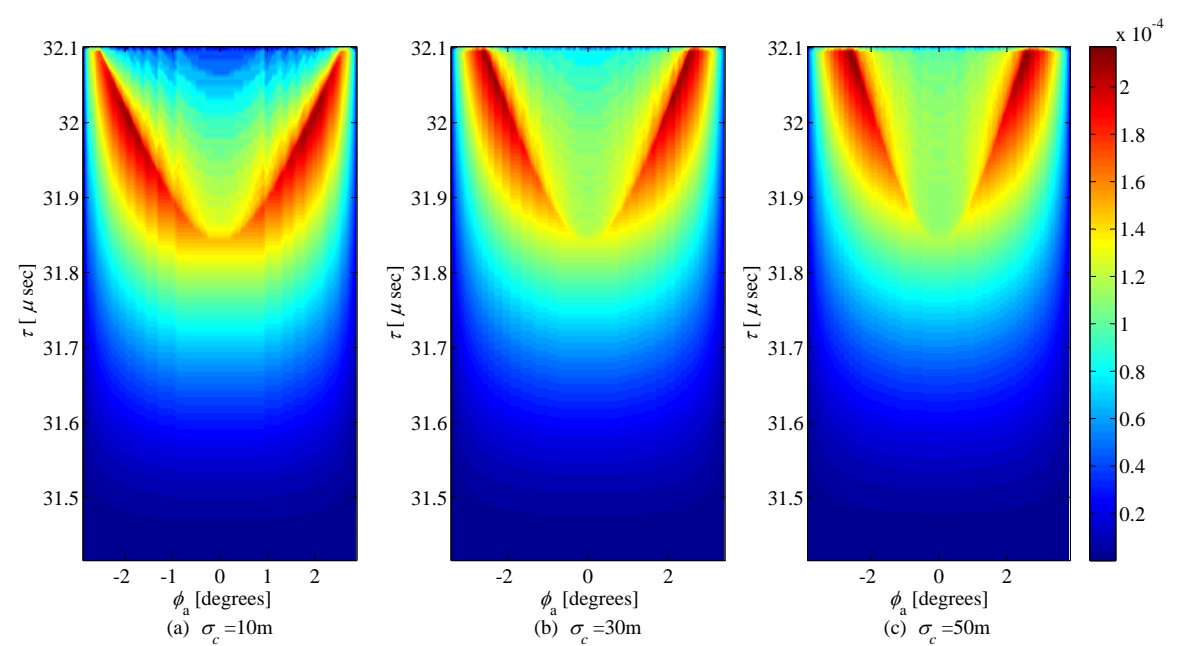

Fig. 11. Joint PDF of ToA and azimuth AoA observed at the AS with GS assumed at ground surface, $\quad\left(h_{\mathrm{AS}}=9 \mathrm{~km}, h_{\mathrm{GS}}=\right.$ $30 \mathrm{~m}, d=3 \mathrm{~km}, \sigma_{t}=0 \mathrm{~m}$, and $\psi_{w}=0 \mathrm{~m}$ ).

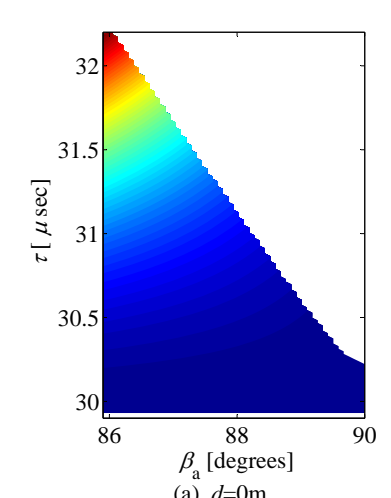

(a) $d=0 \mathrm{~m}$

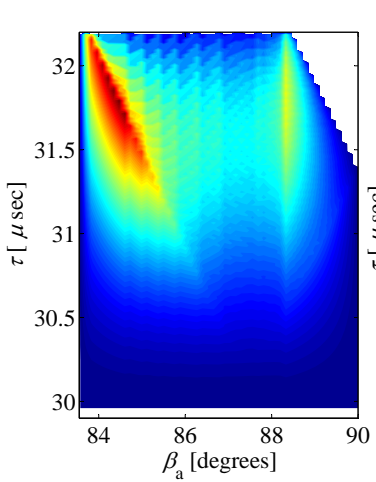

(b) $d=400 \mathrm{~m}$

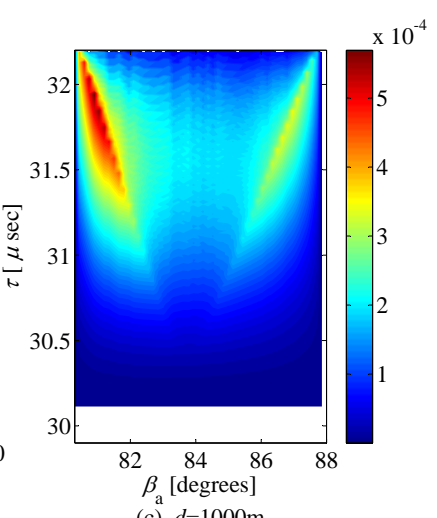

(c) $d=1000 \mathrm{~m}$

Fig. 12. Joint PDF of ToA and elevation AoA observed at the AS with GS assumed at sea surface, $\quad\left(h_{\mathrm{AS}}=9 \mathrm{~km}, h_{\mathrm{GS}}=30 \mathrm{~m}\right.$, $\sigma_{t}=15 \mathrm{~m}, \psi_{w}=5 \mathrm{~m}$, and $\left.\sigma_{c}=15 \mathrm{~m}\right)$.

Fig. 10. The joint ToA and AoA density function seen at AS is similar as those observed from GS due to geometric symmetry except the range of spatial and temporal parameters. The joint PDF of azimuth AoA and ToA seen from AS is shown in (contour) in Fig. 11. This plot is for A2G communication when the GS is taken over ground surface. In A2G radio communication environment, to show the effects of average rooftop level, joint PDF of azimuth AoA and ToA is plotted in Fig. 11 (a), (b), and (c) for $\sigma_{c}$ taken as $10 \mathrm{~m}, 30 \mathrm{~m}$, and 50m, respectively. The joint PDF for ToA with respect to elevation AoA observed from AS is shown (contour) in Fig. 12. This plot is taken for A2G communication when the GS is considered over sea surface (i.e., $\sigma_{d}$ taken as 20m). The effects of distance between AS and GS (i.e., $d$ ) on the joint ToA and elevation AoA statistics can be observed in a Fig. 12 (a), (b), and (c) for $d$ taken as 0m, 400m, and 1000m. The behavior of RMS-DS with respect to the height of AS for different distances between AS 

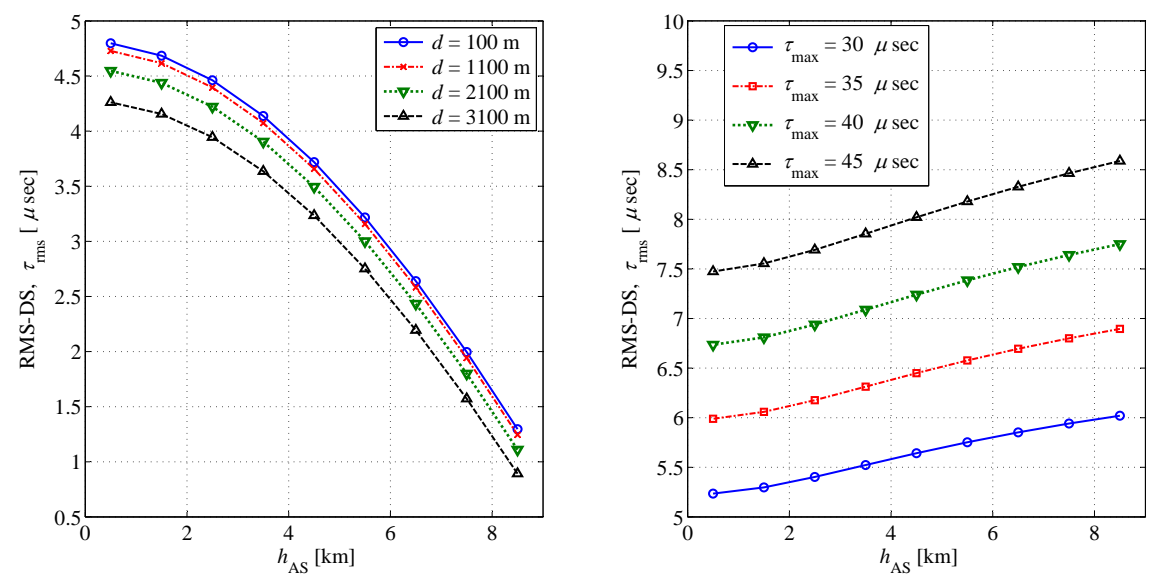

Fig. 13. The behavior of RMS-DS w.r.t height of AS, (a) Impact of horizontal link distance, (b) Impact of channel's delay span. $\left(h_{\mathrm{GS}}=60 \mathrm{~m}, \sigma_{c}=30 \mathrm{~m}\right.$, and $\left.\sigma_{d}=0 \mathrm{~m}\right)$.

and GS and delays of the longest propagation path is shown in Fig.13. It can be seen from Fig.13 (a), that the RMS-DS decreases with increase in AS's height and rate of decreasing increases fastly w.r.t to increase in AS's height and RMS-DS decreases with increase in $d$. It can be seen from Fig.13 (b), that RMS-DS increases with increase in AS's height and longest propagation path's delay. The plots for RMS-DS in Fig.14 are generated for the delay-span $\left(\tau_{\text {span }}=\tau_{\max }-\tau_{o}\right)$ taken at the same range as experimentally measured in [38]. The proposed theoretical results are presented for different values of the link distance, $d=0 \mathrm{~m}, 2900 \mathrm{~m}, 5800 \mathrm{~m}$, and $8700 \mathrm{~m}$. The height of AS and GS are 622m (on average) and 20m, respectively, for the experiments performed in [38]; these parameters are also set the same for the presented theoretical results. Three discrete sample points of the RMS-DS experimental results are plotted in Fig. 14, which are extracted from the experimental data provided in [38] by using graphical data extraction techniques. The mean RMS-DS and average delay-span are measured as $12.9 \mathrm{~ns}$ and $600 \mathrm{~ns}$, respectively, for an A2G communication scenario in [38]. The RMS-DS and delay-span given in [38] is (27ns, 85ns) and (960 ns, $3960 \mathrm{~ns}$ ) for link distances (5735m, 9100m). For the same scenario, a good match between the measured and the proposed theoretical RMS-DS is observed. Thus the validity of model is established.

\section{Conclusions}

A new 3D geometric channel model for A2G/G2A radio communication environments has been proposed. The region of effective scatterers is modeled in 3D space around the GS confined by the 


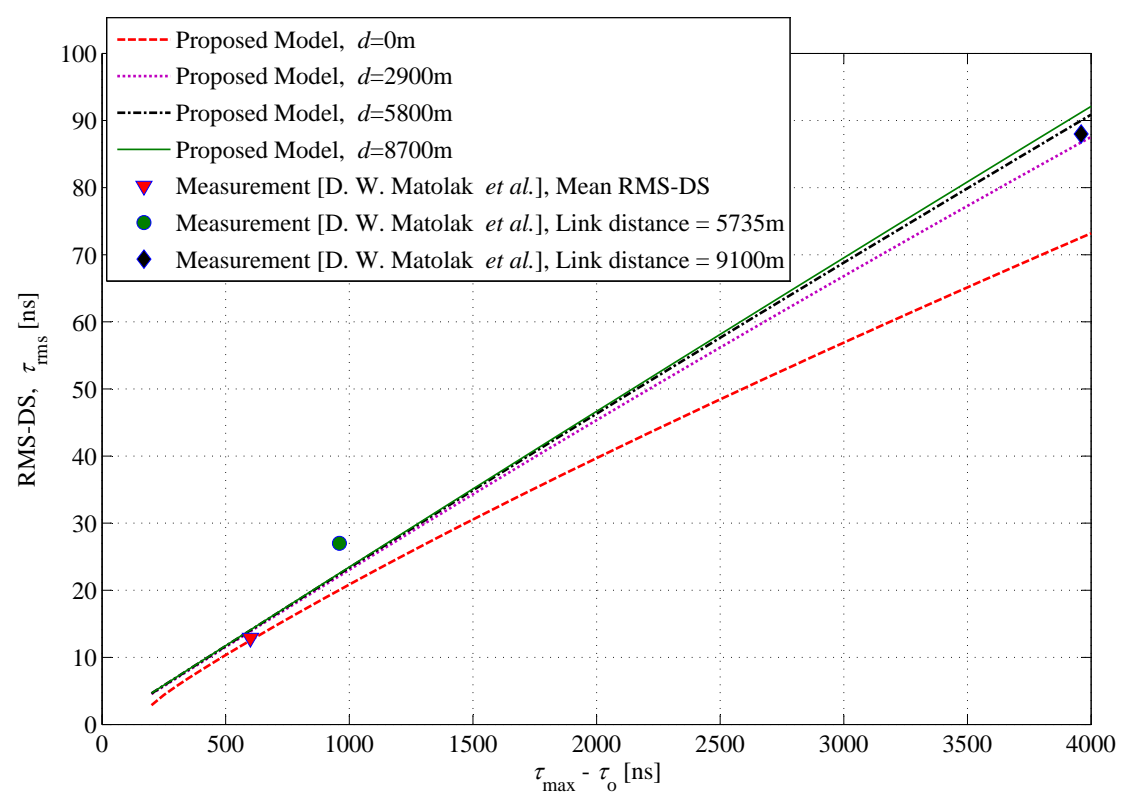

Fig. 14. Comparison of proposed results for RMS-DS with empirical data in Matolak [38] $\left(h_{\mathrm{AS}}=622 \mathrm{~m}, h_{\mathrm{GS}}=20 \mathrm{~m}, \sigma_{c}=10 \mathrm{~m}\right.$, and $\left.\sigma_{d}=0 \mathrm{~m}\right)$.

ellipsoid corresponded by delay longest propagation path and horizontally truncated by the average height of rooftop level in the vicinity of GS. Expressions are derived in close-form for joint and marginal PDFs of AoA and ToA in both azimuth and elevation planes for both up- and downlinks. Analytical results are provided for spatial and temporal characteristics of A2G/G2A channel with comprehensive discussion. On the basis of these theoretical results, it can be established considering the elevation plane in modeling can enhance reliability of the receiver's link at the expense of preprocessing. The impact of variations in the heights of AS and GS on the spatial and temporal channel's characteristics has been studied thoroughly. On the basis of these results, it can be concluded that antenna height can be changed accordingly from the proposed model for a specific propagation environment. The proposed spatial analytical results $A 2 \mathrm{G}$ links are helpful in design of beam-width for phased antenna arrays, for enhancing data rates, as well as a reference for practical data measurement campaigns. Finally, the results of the proposed model are validated by a comparison with simulated results and results presented in a notable model in the literature.

\section{REFERENCES}

[1] G. M. Djuknic, J. Freidenfelds, and Y. Okunev, "Establishing wireless communications services via high altitude platforms: A concept whose time has come?" IEEE Commun. Mag., pp. 128-135, 1997.

[2] M. J. Colella, J. N. Martin, and F. Akyildiz, "The halo network," IEEE Commun. Mag., vol. 38, no. 6, pp. 142-148, Jun. 2000.

[3] R. Janaswamy, "Angle of arrival statistics for a 3-D spheroid model," IEEE Trans. on veh. technol., vol. 51, no. 5, pp. 1242-1247, Sep. 2002. 
[4] J. Zhou, H. Jiang, and H. Kikuchi, "Generalized 3D scattering channel model with MIMO antenna systems," China Commun., vol. 13 , no. 5, pp. 66-81, May.

[5] R. B. Ertel and J. H. Reed, "Angle and time of arrival statistics for circular and elliptical scattering models," IEEE J. on Sel. Areas in Commun., vol. 17, no. 11, pp. 1829-1840, Nov. 1999.

[6] N. M. Khan, M. T. Simsim, and P. B. Rapajic, "A generalized model for the spatial characteristics of the cellular mobile channel," IEEE Trans. on Veh. Technol., vol. 57, no. 1, pp. 22-37, Jan. 2008.

[7] S. J. Nawaz, B. H. Qureshi, and N. M. Khan, "A generalized 3-D scattering model for a macrocell environment with a directional antenna at the BS," IEEE Trans. on Veh. Technol., vol. 59, no. 7, pp. 3193-3204, Sep. 2010.

[8] K. B. Baltzis and J. N. Sahalos, "A simple 3-D geometric channel model for macrocell mobile communication," Wireless Pers. Commun., vol. 51, no. 2, pp. 329-347, Oct. 2009.

[9] P. Petrus, J. H. Reed, and T. S. Rappaport, "Geometrical-based statistical macrocell channel model for mobile environments," IEEE Trans. on Commun., vol. 50, no. 3, pp. 495-502, Mar. 2002.

[10] T. V. Do, N. H. Do, and R. Chakka, "A new queueing model for spectrum renting in mobile cellular networks," Computer Communications, vol. 35, pp. 1165 - 1171, 2012.

[11] M. P. Lotter and P. V. Rooyen, "Modeling spatial aspects of cellular CDMA/SDMA systems," IEEE Commun. Lett., vol. 3, no. 5, pp. 128-131, May 1999.

[12] D. W. Matolak, "Air-ground channels \& models: Comprehensive review and considerations for unmanned aircraft systems," in IEEE Aerosp. Conf., 2012, pp. 1-17.

[13] K. Yang, T. Roste, F. Bekkadal, and T. Ekman, "Channel characterization including path loss and doppler effects with sea reflections for mobile radio propagation over sea at $2 \mathrm{GHz}$," in Int. Conf. on Wireless Commun. and Signal Processing), 2010, pp. 1-6.

[14] E. Haas, "Aeronautical channel modeling," IEEE Trans. on Veh. Technol., vol. 51, no. 2, pp. 254-264, March 2002.

[15] W. G. Newhall, R. Mostafa, C. Dietrich, C. R. Anderson, K. Dietze, G. Joshi, and J. H. Reed, "Wideband air-to-ground radio channel measurements using an antenna array at $2 \mathrm{GHz}$ for low-altitude operations," in Proc. IEEE Military Commun. Conf., vol. 2, 2003, pp. 1422-1427.

[16] Y. S. Meng and Y. H. Lee, "Measurements and characterizations of air-to-ground channel over sea surface at C-band with low airborne altitudes," IEEE Trans. on Veh. Technol., vol. 60, no. 4, pp. 1943-1948, May 2011.

[17] Y. H. Lee, Y. S. Meng, and Y. H. Heng, "Experimental characterizations of an air to land channel over sea surface in C band," in Proc. XXIXth URSI General Assembly, Chicago, Illinois, USA, Aug. 2008.

[18] M. Rice, A. Davis, and C. Bettweiser, "Wideband channel model for aeronautical telemetry," IEEE Trans. on Aerosp. and Electron. Syst., vol. 40, no. 1, pp. 57-69, Jan. 2004.

[19] P. A. Bello, "Aeronautical channel characterization," IEEE Trans. on Commun., vol. COM-21, no. 5, pp. 548-563, May 1973.

[20] Q. Lei and M. Rice, "Multipath channel model for over-water aeronautical telemetry," IEEE Trans. on Aerosp. and Electron. Syst., vol. 45, no. 2, pp. 735-742, Apr. 2009.

[21] T. Willink, C. Squires, G. Colman, and M. Muccio, "Measurement and characterization of low altitude air-to-ground MIMO channels," IEEE Trans. on Veh. Technol., vol. PP, no. 99, pp. 1-1, 2015.

[22] D. Medina and F. Hoffmann, The Airborne Internet. INTECH Open Access Publisher, 2011.

[23] S. J. Nawaz, N. M. Khan, M. I. Tiwana, N. Hassan, and S. I. Shah, "Airborne internet access through submarine optical fiber cables," IEEE Trans. on Aerosp. and Electron. Syst., vol. 51, no. 1, pp. 167-177, Jan. 2015.

[24] A. Mohammed, A. Mehmood, F. N. Pavlidou, and M. Mohorcic, "The role of high-altitude platforms (haps) in the global wireless connectivity," Proceedings of the IEEE, vol. 99, no. 11, pp. 1939-1953, Nov. 2011.

[25] F. Dovis, R. Fantini, M. Mondin, and P. Savi, " $4 \mathrm{~g}$ communications based on high altitude stratospheric platforms: channel modeling and performance evaluation,” in IEEE Global Telecommun. Conf., GLOBECOM '01., vol. 1, 2001, pp. 557-561 vol.1.

[26] _ - "Small-scale fading for high-altitude platform (hap) propagation channels," IEEE Journal on Sel. Areas in Commun., vol. 20, no. 3, pp. 641-647, Apr. 2002.

[27] E. T. Michailidis and A. G. Kanatas, "Three-dimensional hap-mimo channels: Modeling and analysis of space-time correlation,” IEEE Trans. on veh. technol., vol. 59, no. 5, pp. 2232-2242, Jun. 2010.

[28] J. C. Liberti and T. S. Rappaport, "A geometrically based model for line of sight multipath radio channels," in Proc. IEEE 46th Veh. Technol. Conf., vol. 2, Apr. 1996, pp. 844-848.

[29] X. Gao, Z. Chen, and Y. Hu, "Analysis of unmanned aerial vehicle MIMO channel capacity based on aircraft attitude," WSEAS Trans. on Informa. Sc. and App., vol. 10, no. 2, pp. 58-67, Feb. 2013.

[30] M. Walter and M. Schnell, "The doppler-delay characteristic of the aeronautical scatter channel," in proc. of IEEE Veh. Technol. Conf., Sep. 2011, pp. 1-5.

[31] J. H. Painter, S. C. Gupta, and L. Wilson, "Multipath modeling for aeronautical communications," IEEE Trans. on Commun., vol. 21 , no. 5, pp. 658-662, 1973.

[32] W. G. Newhall and J. H. Reed, "A geometric air-to-ground radio channel model," in Proc. IEEE Military Commun. Conf., Oct. 2002, pp. 632-636.

[33] B. Zheng, Q. H. Ren, Y. J. Liu, , and Z. Y. Chu, "Simulation of two V/UHF air-to-ground communication channel models," in Proc. Int. Conf. on Wireless Commun., Networking and Mobile Computing, 2007, pp. 1083-1086.

[34] M. Riaz, S. J. Nawaz, and N. M. Khan, “3D ellipsoidal model for mobile-to-mobile radio propagation environments," Wireless Pers. Commun., vol. 72, pp. 2465-2479, Apr. 2013.

[35] S. M. Gulfam, S. J. Nawaz, M. N. Patwary, and M. Abdel-Maguid, "On the spatial characterization of 3-D air-to-ground radio communication channels,” in Proc. of IEEE Int. Conf. on Commun. (ICC), Jun. 2015, pp. 2924-2930. 
[36] S. Jiang and S. Georgakopoulos, "Electromagnetic wave propagation into fresh water," Journal of Electromagnetic Analysis \& Applications, vol. 3, no. 7, 2011.

[37] K. B. Baltzis, "A generalized elliptical scattering model for the spatial characteristics of mobile channels," Wireless Personal Communications, vol. 67, no. 4, pp. 971-984, 2012.

[38] D. W. Matolak and R. Sun, "Air-ground channel characterization for unmanned aircraft systems: the hilly suburban environment," in 80th IEEE Veh. Technol. Conf., 2014, pp. 1-5. 